\title{
Optimal control of the cylinder wake in the laminar regime by Trust-Region methods and POD Reduced-Order Models
}

\author{
M. Bergmann ${ }^{\mathrm{a}, *}$, L. Cordier ${ }^{\mathrm{b}}$ \\ ${ }^{a}$ Institut de Mathématiques de Bordeaux, UMR 5251 CNRS, Université Bordeaux \\ 1 and INRIA Bordeaux Sud Ouest MC2, 351, Cours de la Libération, 33405 \\ Talence cedex, France \\ ${ }^{\mathrm{b}}$ Laboratoire d'Etudes Aérodynamiques (Université de Poitiers, ENSMA, CNRS) \\ 43, rue de l'Aérodrome 86036 Poitiers cedex, France.
}

\begin{abstract}
In this paper, optimal control theory is used to minimize the total mean drag for a circular cylinder wake flow in the laminar regime $(R e=200)$. The control parameters are the amplitude and the frequency of the time-harmonic cylinder rotation. In order to reduce the size of the discretized optimality system, a Proper Orthogonal Decomposition (POD) Reduced-Order Model (ROM) is derived to be used as state equation. We then propose to employ the Trust-Region Proper Orthogonal Decomposition (TRPOD) approach, originally introduced by Fahl (2000), to update the reduced-order models during the optimization process. A lot of computational work is saved because the optimization process is now based only on low-fidelity models. A particular care was taken to derive a POD ROM for the pressure and velocity fields with an appropriate balance between model accuracy and robustness. The key enablers are the extension of the POD basis functions to the pressure data, the use of calibration methods for the POD ROM and the addition in the POD expansion of several non-equilibrium modes to describe various operating conditions. When the TRPOD algorithm is applied to the wake flow configuration, this approach converges to the minimum predicted by an open-loop control approach and leads to a relative mean drag reduction of $30 \%$ at reduced cost.
\end{abstract}

Key words: Model reduction, Proper Orthogonal Decomposition, Surrogate optimization, Adaptive strategy

\footnotetext{
* Corresponding author.

Email addresses: Michel.Bergmann@inria.fr (M. Bergmann), Laurent.Cordier@univ-poitiers.fr (L. Cordier).
} 


\section{Introduction}

\subsection{Reduced-order models in optimization}

During the last decade, the optimal control theory [1] has emerged as a new approach to solve active flow control and aerodynamic shape design problems. Indeed, these problems can be reduced [2] to the minimization or maximization of an objective functional $\mathcal{J}$ according to $n$ control or design parameters $\boldsymbol{c}=\left(c_{1}, c_{2}, \cdots, c_{n}\right)$ under some constraints. However, whatever the specific class of numerical methods generally considered (methods of descent type, stochastic methods), the computational costs (CPU and memory) related to the resolution of optimization problems are so important that they become unsuited to the applications of flow control for three-dimensional turbulent flows. The application in an immediate future of active control to complex flows is thus conditioned by the development of approximate models of the system [3]. The objective of these surrogate models [4] is to capture the essence of the physics of the controlled system while reducing the costs associated to the solution of the nonlinear state equations. As a result, there have been many studies devoted to the development of Reduced-Order Models (ROM) that serve as low-dimensional approximation models to the large-scale discretized Navier-Stokes equations [5, for a review of the different reduced-order modelling techniques]. The model reduction method discussed in this paper fall in the category of reduced basis approaches. For the reduced bases, a number of choices exist [6, for a presentation] : Lagrange basis, Hermite basis, Taylor basis, Proper Orthogonal Decomposition (POD) basis [7,8], Krylov basis [9], Centroidal Voronoi Tessellations (CVT) basis [10], balanced POD basis [11], etc. Today, the most popular reduced-order modelling approach for complex systems in fluid mechanics is based on POD. This study is restricted to this case: we consider that the unsteady non-linear dynamics of the flow is modelled via a reduced-order model based on POD (POD ROM).

The POD (and other similar techniques of ROM) can be viewed as a method of information compression. Essentially, the POD algorithm try to remove "redundant" information (if any) from the data base. As a consequence, the ability of POD modes to approximate any state of a complex system is totally dependent of the information originally contained in the snapshot set used to generate the POD functions. Thus, a POD basis cannot contain more information than that contained in the snapshot set. The generation of "good" snapshot set is then crucial to the success of use of POD ROM approach in a bifurcation analysis [12-14] or more generally in an optimization setting. Since the POD basis is intrinsic to a particular flow, we need to give special attention to adapt the POD ROM (and the POD basis naturally) to changes in physics when the flow is altered by control. This central question is discussed 
in more details in [15] where two strategies are evidenced for use of POD ROM in an optimization setting. A first approach consists in distributing uniformly in the control parameter space the snapshot ensemble to be used for POD. However, in this case, a lot of runs of the high-dimensional code would be necessary to generate the snapshots and that more especially as the number of the control parameters is important. Therefore, developing systematic and rational methodologies for generating good snapshots set is a critical enabler for effective reduced-order modelling, since a POD basis is only as good as the snapshot set used to generate it. Very recently, it was demonstrated in [10] that Centroidal Voronoi Tessellations could be one method of intelligent sampling in parameter space. Failing this, a simpler method to implement is to generate generalized POD functions by forcing the flow with an ad-hoc time-dependent excitation that is rich in transients [16]. The second approach consists of an adaptive method in which new snapshots are regularly determined during the optimization process when the effectiveness of the existing POD ROM to represent accurately the controlled flow is considered to be insufficient [17-19]. At this point, two key questions still remain:

(1) How to decide automatically whether or not a POD ROM has to be adapted to a new flow configuration?

(2) Can we demonstrate under certain conditions (which should ideally be most general as possible) that the optimal solution based on the POD ROM corresponds to a local optimizer for the original problem?

The main drawback of this second approach is that for adaptively updating a reduced basis during an optimization process, new solves of the highdimensional approximations of the Navier-Stokes equations need to be done. Since these simulations are costly, this approach is not appropriate for realtime control flow.

\subsection{A generic configuration of separated flows: the cylinder wake flow}

Due to its simple geometry and its representative behavior of separated flows [20], the cylinder wake flow has been broadly studied this past decade to experiment some control methods that could be used later in more complex engineering configurations. The majority of these studies were motivated by the experiments of [21] where $80 \%$ of relative ${ }^{1}$ mean drag reduction was empirically found at $R e=15,000$ by unsteady rotary oscillation of the cylinder. This

\footnotetext{
${ }^{1}$ Here, and in the following occurrences in the text, the relative mean drag reduction is defined as $\left(\left\langle C_{D}\right\rangle_{T}^{\text {unforced }}-\left\langle C_{D}\right\rangle_{T}^{\text {forced }}\right) /\left\langle C_{D}\right\rangle_{T}^{\text {unforced }}$ where $\left\langle C_{D}\right\rangle_{T}$, the mean temporal drag coefficient estimated over a finite horizon $T$, is defined in Eq. (3). The terms 'unforced' and 'forced' are used respectively for non-rotating and rotating cylinder.
} 
experimental work was followed by a series of numerical [22-30] and experimental investigations [31-34]. Recently, due to the maturity of control theory, optimization methods and computational fluid dynamics, optimal and suboptimal approaches attracted increased attention in flow control setting [35-37]. For example, in [38-40] the optimal control theory was used with the twodimensional Navier-Stokes equations as the state equation to control by rotary oscillation the unsteady wake of the cylinder (see table 1 for the characteristics of these approaches). An attractive element of the optimal control approach is that the control design is explicitly based on the cost functional. However, the very large computational costs (CPU and memory), involved in the resolution of the optimality system commonly used in the optimal control theory [2], prevent to solve routinely optimization problems based on the three-dimensional Navier-Stokes equations ${ }^{2}$. For cutting down these numerical costs different approaches are possible [3, for a review]. One promising approach is to first develop POD ROM to approximate the fluid flow and then to optimize exactly the reduced-order models as it was already discussed in section 1.1. A general discussion of the use of approximation models in optimization can be found in [44]. In this study, we want to develop a low-cost optimal control approach for the drag minimization of the cylinder wake with rotary motion for control law (see Fig. 1). In addition, as opposed to what was made in [15], where the cost functional to be minimized was not the drag but a drag-related cost function (the turbulent kinetic energy contained in the wake), we will directly take here for cost functional the mean drag coefficient (viscous and pressure contributions). Then, to reduce as much as possible the computational costs associated to the present study, the flow is considered two-dimensional and in the laminar regime. However, the methodology presented here that consists of combining the optimal control approach and a POD ROM should easily be expanded to three-dimensional and turbulent flows.

In their numerical investigation of the controlled wake flow by rotary oscillation of the cylinder, Protas and Wesfreid [30] argued that in the supercritical regime, the effectiveness of the control in terms of drag reduction increases with the Reynolds number. This important result was recently confirmed by a study of our group [45] which showed analytically that the power necessary to control the wake by unsteady rotation varied, for fixed values of the control parameters $A$ and $S t_{f}$, like the inverse of the square root of the Reynolds number. Therefore, since the wake flow remains two-dimensional up to a value of the Reynolds number approximately equal to 190 where a spanwise supercritical Hopf bifurcation occurs and where the three-dimensional effects appear

2 Two exceptions are the seminal work of [41] and the subsequent study of [42] where the optimal control theory is used to determine controls that reduce the drag of a turbulent flow in a three-dimensional plane channel simulated at $R e_{\tau}=180$. More recently, an optimal control approach was used in [43] to reduce the sound generated by a two-dimensional mixing layer. 
[46,47], the "optimal" value of the Reynolds number for our two-dimensional study is slightly lower than 200. However for facilitating the comparisons with the results of the literature, a Reynolds number of 200 is considered. According to the observations of [38], the control minimizing the drag generates vortices that are less energetic than those produced by the stationary cylinder. An energetic criterion seems to be well adapted to the investigation of drag reduction. Therefore, due to the energetic optimality of convergence of the POD basis $[7,8,48]$, the choice of POD to develop a reduced-order model of the controlled unsteady flow seems to be well adapted. A similar approach was already considered in $[16,49]$ to control the wake flow at a supercritical Reynolds number of 100.

Finally, we need to choose between the two opposite strategies discussed at the end of section 1.1. If we want to develop active flow control method that can be used for real-time, on-line feedback control, our interest is to include in the snapshot set all the information needed during the optimization process or at least as much information as we can, and then to generate the reducedorder basis. Following this approach the POD functions are determined once for all at the beginning of the optimization process and no refresh is realized. This method was successfully applied to control the cylinder wake flow in [15]. It was demonstrated that an accurate and robust POD ROM can be derived using a snapshot ensemble for POD based on chirp-forced transients of the flow. Moreover, $25 \%$ of relative drag reduction was found when the NavierStokes equations were controlled using an harmonic control law deduced from the optimal solution determined with the POD ROM. However, the excitation used to determine the generalized POD functions lacks of justifications and, with this particular approach, there is no mathematical assurance that the optimal solution based on the POD ROM corresponds to a local optimizer for the high-fidelity model. The same remark can be made concerning the approaches presented in $[17,18]$ and $[19]$. Indeed, in these articles, a new POD ROM is determined when the control law does not evolve sufficiently with the previous model. With this strategy, there is not any proof that the control which is finally obtained is solution of the initial problem of optimization. Therefore, in this paper, we propose to use a specific adaptive method called Trust-Region Proper Orthogonal Decomposition (TRPOD) to update the reduced-order models during the optimization process. This approach, originally introduced by [50], benefits from the trust-region philosophy [51, for an introduction]. Then, rigorous convergence results guarantee that the iterates produced by the TRPOD algorithm will converge to the solution of the original optimization problem defined with the Navier-Stokes equations, the 
so-called global convergence ${ }^{3}$ of the trust-region methods. Moreover, in [15], the POD basis used to derive the reduced-order models represented only velocities. Therefore, a drag-related cost functional characteristic of the wake unsteadiness was minimized. Since the pressure term contributes to approximately $80 \%$ of the total drag coefficient for a Reynolds number equal to 200, here, a pressure POD basis was determined ( $\S 4.1$ ), allowing us to consider the total drag as objective functional in our optimal control approach $(\S 4.3)$.

The main objective of this paper is to demonstrate in a simple flow control configuration that the use of the TRPOD algorithm can be successful to determine at least a local minimizer of the original problem. To supplement this main result, we will also give an estimate of the computational savings that can be obtained by a POD ROM based optimal control approach compared with the more "classical" approach where the Navier-Stokes equations are used for constraints [38-40]. Consequently in this study our main concern is not to determine the control law with the maximum energetic efficiency as it can be characterized for example by the Power Saving Ratio (PSR) [40, for a definition or hereafter in $\S 5.2 .3]$. As far as we know (see table 1), the work presented in [40] is the only one which considers for cost functional the sum of the drag power and the control power thus making it possible to determine an optimal solution that is by construction energetically efficient. In the other works, the cost of the control is not considered or at best as a regularization parameter. This discussion will be developed in section 5.2.3 where we compare the energetic efficiency of the different approaches.

This manuscript is organized as follows. Section 2 begins with the introduction of the generic controlled flow configuration. In the next two subsections, a mathematical expression of the mean drag coefficient is first introduced (§ 2.2), then an open-loop control study of the cylinder wake is carried out (§ 2.3). The optimization by Trust-Region methods and POD Reduced-Order Models is presented in $\S 3$ where the Trust-Region POD (TRPOD) algorithm is formally introduced. Following the philosophy of trust-region methods, a robust surrogate function for the mean drag coefficient is then constructed in $\S 4$. The key enablers are the extension of the POD basis to the pressure field $(\S 4.1)$ and the introduction of non-equilibrium modes in the POD expansion to represent different operating conditions $(\S 4.2)$. Finally, we formulate an optimal control problem for the POD ROM ( $§ 5.1)$ and present the numerical results of the mean drag minimization of the cylinder wake flow obtained by a suboptimal and an optimal (TRPOD) POD-based adaptive controllers ( $§ 5.2)$.

$\overline{3}$ Let us consider a general unconstrained optimization problem $\min _{\boldsymbol{c} \in \mathbb{R}^{n}} f(\boldsymbol{c})$. The global convergence result of the trust-region methods states [50, for example] that $\lim _{k \longrightarrow \infty}\left\|\nabla f\left(\boldsymbol{c}_{k}\right)\right\|=0$ where $k$ represents the index of a current iterate of the iterative method. 


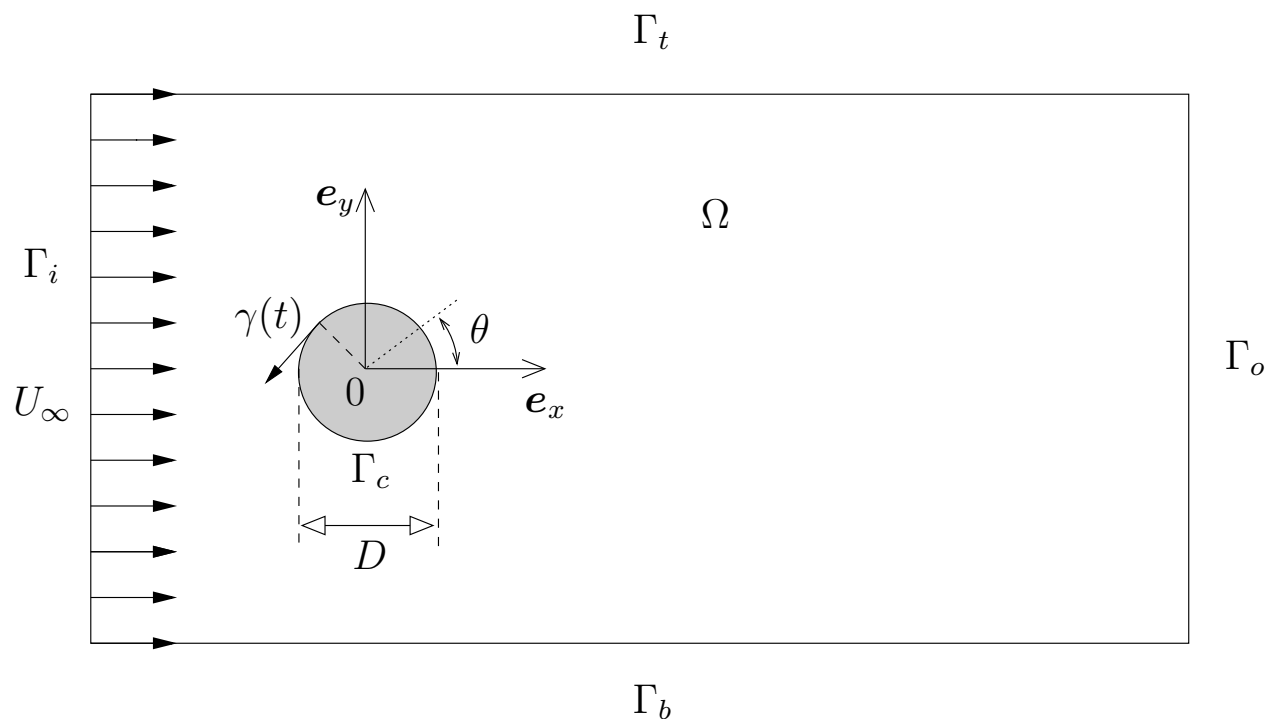

Fig. 1. Controlled flow configuration.

\section{Problem formulation}

\subsection{Flow configuration and governing equations}

Let $\Omega$ be a two-dimensional bounded region filled with a Newtonian incompressible viscous fluid of kinematic viscosity $\nu$ and $\Gamma$ denote the boundaries of $\Omega$ (Fig. 1). The velocity vector is $\boldsymbol{u}=(u, v)$, where $u$ and $v$ are the components in the $\boldsymbol{e}_{x}$ and $\boldsymbol{e}_{y}$ direction, respectively. Pressure is denoted by $p$. In the following, all variables are assumed to be non-dimensionalized with respect to the cylinder diameter $D$ ( $R$ is the corresponding radius) and the uniform velocity of the incoming flow $U_{\infty}$. $[0, T]$ corresponds to the time interval during which the flow is considered.

Mathematically, the problem can be described by the incompressibility condition and the two-dimensional unsteady Navier-Stokes equations:

$$
\left\{\begin{array}{l}
\boldsymbol{\nabla} \cdot \boldsymbol{u}=0 \quad \text { in } \Omega \times[0, T] \\
\frac{\partial \boldsymbol{u}}{\partial t}+\nabla \cdot(\boldsymbol{u} \otimes \boldsymbol{u})=-\nabla p+\frac{1}{R e} \boldsymbol{\Delta} \boldsymbol{u} \quad \text { in } \Omega \times[0, T]
\end{array}\right.
$$

where $R e=U_{\infty} D / \nu$ is the Reynolds number.

The objective of this study is the mean drag minimization of the wake flow by rotary oscillation of the cylinder as in the experiments of [21]. The rotary control will be characterized by the instantaneous tangential velocity $\gamma(t)$. In [15], no particular assumption was done on the variation of the control 
law, $\gamma$. Here, $\gamma(t)$ is sought using the optimal control theory as an harmonic function of the form:

$$
\gamma(t)=A \sin \left(2 \pi S t_{f} t\right)
$$

where $A$ and $S t_{f}=f_{f} D / U_{\infty}$ are the amplitude and the Strouhal number of the forcing, respectively. Finally, for later notations convenience, we introduce the control vector $\boldsymbol{c}=\left(A, S t_{f}\right)^{T}$.

The same Navier-Stokes solver as in [15] was used for this study. The reader is thus refered to [15] or [52] for a description of the numerical methods.

\subsection{Mean drag coefficient}

In a viscous flow the total forces acting on a body are contributed by the pressure and skin friction terms. For a circular cylinder, the instantaneous drag coefficient $C_{D}$ is given by $C_{D}(t)=\mathcal{C}_{\mathcal{D}}(\boldsymbol{U})$ where $\boldsymbol{U}=(u, v, p)^{T}=\left(\boldsymbol{u}^{T}, p\right)^{T}$ is the vector corresponding to the velocity and pressure fields obtained as solutions of the Navier-Stokes equations $(1)$, and where $\mathcal{C}_{\mathcal{D}}$ is the drag operator defined for any given vector $\boldsymbol{b}=\left(b_{1}, b_{2}, b_{3}\right)^{T}$ in $\mathbb{R}^{3}$ as:

$$
\begin{aligned}
\mathcal{C}_{\mathcal{D}}: \mathbb{R}^{3} & \rightarrow \mathbb{R} \\
\boldsymbol{b} & \mapsto 2 \int_{0}^{2 \pi}\left(b_{3} n_{x}-\frac{1}{R e} \frac{\partial b_{1}}{\partial x} n_{x}-\frac{1}{R e} \frac{\partial b_{1}}{\partial y} n_{y}\right) R \mathrm{~d} \theta .
\end{aligned}
$$

In (2), $n_{x}$ and $n_{y}$ are the projections of $\boldsymbol{n}$, the external normal vector to the boundary $\Gamma_{c}$, onto the cartesian basis vectors $\boldsymbol{e}_{x}$ and $\boldsymbol{e}_{y}$ respectively, and $\theta$ is an angle defining the curvilinear coordinate of a point on $\Gamma_{c}$. By convention, this angle is initialized at the front stagnation point of the cylinder (see Fig. 1).

For the optimal control procedure, the objective function will correspond to the mean time drag coefficient estimated over a finite horizon $T$ equal to a few vortex shedding periods, i.e.

$$
\mathcal{J}(\boldsymbol{U})=\left\langle C_{D}(t)\right\rangle_{T}=\left\langle\mathcal{C}_{\mathcal{D}}(\boldsymbol{U})\right\rangle_{T}=\frac{1}{T} \int_{0}^{T} \mathcal{C}_{\mathcal{D}}(\boldsymbol{U}) \mathrm{d} t
$$

\subsection{Open-loop control of the cylinder wake}

The main results of an open-loop control study performed numerically at $R e=200$ to validate a posteriori the control law obtained with the POD based optimization method are now summarized. In order to analyze the influence of the forcing parameters $A$ and $S t_{f}$ onto the mean drag coefficient, a 


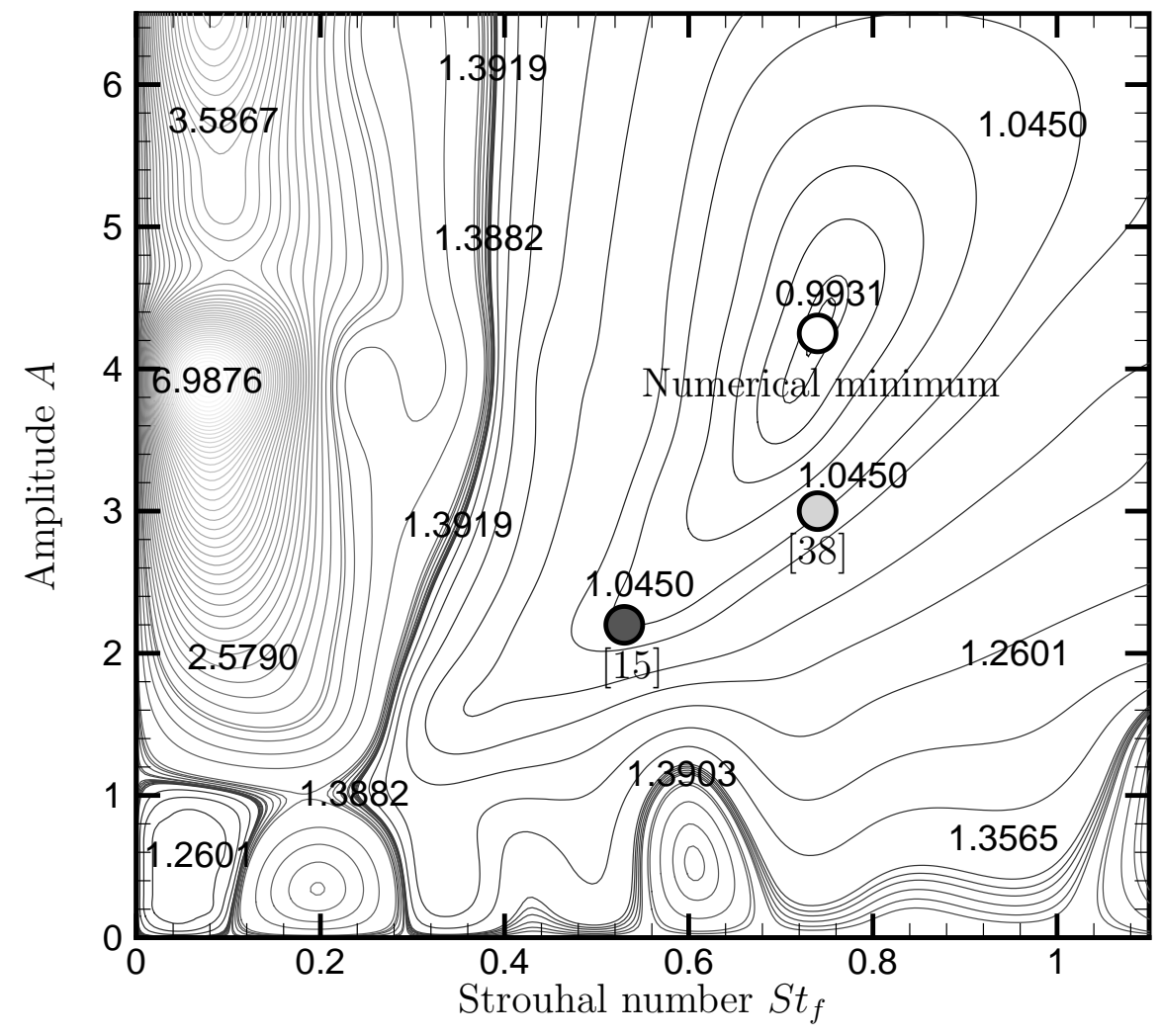

Fig. 2. Variation of the mean drag coefficient with $A$ and $S t_{f}$ at $R e=200$. Numerical minimum: $\left(A_{\min }, S t_{f_{\min }}\right)=(4.25,0.74) ;[38]:\left(A, S t_{f}\right)=(3 ., 0.75) ;[39]$ (not shown): $\left(A, S t_{f}\right)=(3.25,1.13) ;[15]:\left(A, S t_{f}\right)=(2.2,0.53)$.

series of simulations with different amplitude $A$ varying from 0 to 6.5 by step of 0.5 and different forcing Strouhal number $S t_{f}$ varying from 0 to 1 . by step of 0.1 was made. For a Reynolds number equal to 200, the forcing frequency $S t_{f}$ ranges from one-half to five natural shedding frequency $S t_{n}$. For every forcing frequency our simulations are performed for a sufficient long time $\left(T_{S}=130\right)$ to assure that the saturated state has been reached. All simulations have been done with the same time step, here equal to $1.510^{-2}$. In Fig. 2, we visualize the contours of the mean temporal drag estimated over the last 30 units of time in the space spanned by the forcing parameters $A$ and $S t_{f}$. In this figure, interpolations by spline functions were done between the values of mean drag coefficient obtained for the various control parameters. Numerically, the mean drag coefficient reaches a minimal value, which appears to correspond to a global minimum of the drag function on the domain studied, for an optimal pair $\left(A_{\min }, S t_{f_{\min }}\right)=(4.25,0.74)$. The corresponding minimum value is 0.99 . It is noticeable that the function defined by the mean drag coefficient is rather regular, and that the minimum is located in a smooth valley. Another striking 
feature of this open-loop control study is that with the use of a sinusoidal control law on the whole cylinder the minimal value of the mean drag coefficient remains greater than that obtained for the basic flow $\left(\left\langle C_{D}^{\text {basic }}\right\rangle_{T}=0.94\right.$ at $R e=200$, see [15]) as it was argued by [30]. In other words, the value of the mean drag correction term is always positive.

\section{Optimization by Trust-Region methods and POD Reduced-Order Models}

The philosophy of combining trust-region methods with approximation models of different level of reliability is a well known technique in multidisciplinary design optimization that is named surrogate optimization [44]. In the spirit of this approach, the Trust-Region Proper Orthogonal Decomposition (TRPOD) was recently proposed in [50] and [53] as a way to overcome the main difficulties related to the use of a POD ROM to solve an optimization problem. First, when the POD technique is embedded into the concept of trust-region frameworks with general model functions (see [54] for a comprehensive survey or [51] for an introduction on trust-region methods) a mechanism is provided to decide when an update of the POD ROM is necessary during the optimization process. Second, from a theoretical point of view, global convergence results exist [50] that prove that the iterates produced by the optimization algorithm, started at an arbitrary initial iterate, will converge to a local optimizer for the original model.

Hereafter, we consider that the flow control problem (minimization of the mean drag coefficient for example) can be formulated as an unconstrained optimization problem

$$
\min _{\boldsymbol{c} \in \mathbb{R}^{n}} \mathcal{J}\left(\boldsymbol{\zeta}_{\mathrm{NS}}(\boldsymbol{c}), \boldsymbol{c}\right)
$$

where $\mathcal{J}: \mathbb{R}^{m} \times \mathbb{R}^{n} \mapsto \mathbb{R}$ represents the objective function and where $\boldsymbol{\zeta}_{\mathrm{NS}}$ and $\boldsymbol{c}$ respectively represent the state variables obtained by numerical resolution of the state equations and the control variables. The subscript NS means that the state equations which connect the control variables $\boldsymbol{c}$ to the state variables are the Navier-Stokes equations.

Since an accurate computation of the state variables $\boldsymbol{\zeta}$ for given $\boldsymbol{c}$ is computationally expensive when the Navier-Stokes equations are used as the state equations, the evaluation of $\mathcal{J}$ during the solution of the optimization process (4) is computationally expensive. A reduction of numerical cost can be achieved by employing a POD ROM as the state equation. In such a way an approximate solution $\boldsymbol{\zeta}_{\mathrm{POD}}$ of the state variables $\boldsymbol{\zeta}$ is obtained and the optimization problem (4) is then replaced by a succession of subproblems of the form 


$$
\min _{\boldsymbol{c} \in \mathbb{R}^{n}} \mathcal{J}\left(\boldsymbol{\zeta}_{\mathrm{POD}}(\boldsymbol{c}), \boldsymbol{c}\right)
$$

Usually, a POD ROM is constructed for a specific flow configuration, e.g., for an uncontrolled flow or for a flow altered by a specified control. Therefore, the range of validity of a given POD ROM is generally restricted to a region located in the vicinity of the design parameters in the control parameter space, the so-called trust-region. It is then necessary to update the POD ROM during the iterative process, the crucial point being to determine when such a reactualization must take place.

Let $\Delta^{(k)}>0$ be the trust-region radius and $\boldsymbol{c}^{(k)}$ be the control vector obtained at an iterate $k$ of the optimization process. To evaluate the function $\mathcal{J}\left(\boldsymbol{\zeta}_{\mathrm{NS}}\left(\boldsymbol{c}^{(k)}\right), \boldsymbol{c}^{(k)}\right)$, it is necessary to determine the state variables $\boldsymbol{\zeta}_{\mathrm{NS}}\left(\boldsymbol{c}^{(k)}\right)$. These variables are obtained by resolution of the high-fidelity model, the Navier-Stokes equations solved with $\boldsymbol{c}=\boldsymbol{c}^{(k)}$. Then, we compute snapshots that correspond to the flow dynamics forced by $\boldsymbol{c}^{(k)}$. These snapshots form the input ensemble necessary to generate a POD basis $\left\{\boldsymbol{\phi}_{i}^{(k)}\right\}_{i=1, \ldots, N_{\mathrm{POD}}}$. This POD basis can then be used via a Galerkin projection of the Navier-Stokes equation onto the POD eigenvectors to derive a POD ROM for $\boldsymbol{c}^{(k)}$ (see $\S$ 4.1.2). After integration in time of this POD ROM, the state variables $\boldsymbol{\zeta}_{\mathrm{POD}}\left(\boldsymbol{c}^{(k)}\right)$ are estimated, and thus the function $\mathcal{J}\left(\boldsymbol{\zeta}_{\mathrm{POD}}\left(\boldsymbol{c}^{(k)}\right), \boldsymbol{c}^{(k)}\right)$ is evaluated. Since this POD ROM can be employed for an optimization cycle, we define

$$
m^{(k)}\left(\boldsymbol{c}^{(k)}+\boldsymbol{s}^{(k)}\right)=\mathcal{J}\left(\boldsymbol{\zeta}_{\mathrm{POD}}\left(\boldsymbol{c}^{(k)}+\boldsymbol{s}^{(k)}\right), \boldsymbol{c}^{(k)}+\boldsymbol{s}^{(k)}\right),
$$

as a model function for

$$
f\left(\boldsymbol{c}^{(k)}+\boldsymbol{s}^{(k)}\right)=\mathcal{J}\left(\boldsymbol{\zeta}_{\mathrm{NS}}\left(\boldsymbol{c}^{(k)}+\boldsymbol{s}^{(k)}\right), \boldsymbol{c}^{(k)}+\boldsymbol{s}^{(k)}\right),
$$

on the trust-region $\left\|\boldsymbol{s}^{(k)}\right\| \leq \Delta^{(k)}$ around $\boldsymbol{c}^{(k)}$.

One is then brought to solve the corresponding trust-region subproblem defined as

$$
\min _{\boldsymbol{s} \in \mathbb{R}^{n}} m^{(k)}\left(\boldsymbol{c}^{(k)}+\boldsymbol{s}\right), \quad \text { s.t. }\|\boldsymbol{s}\| \leq \Delta^{(k)}
$$

Following the trust-region philosophy [54], it is not necessary to determine the exact step solution of the problem (8). It is sufficient to compute a trial step $\boldsymbol{s}^{(k)}$ that achieves only a certain amount of decrease for the model function [55]. However, due to the low computational costs involved to solve the reducedorder model, the problem (8) can be solved exactly (see the optimal control formulation described in section 5.1). 
In order to estimate the quality of the presumed next control parameters $\boldsymbol{c}^{(k+1)}=\boldsymbol{c}^{(k)}+\boldsymbol{s}^{(k)}$ where $\boldsymbol{s}^{(k)}$ is the solution of (8), we compare the actual reduction in the true objective, $f\left(\boldsymbol{c}^{(k+1)}\right)-f\left(\boldsymbol{c}^{(k)}\right)$, to the predicted reduction obtained with the model function $m^{(k)}\left(\boldsymbol{c}^{(k+1)}\right)-m^{(k)}\left(\boldsymbol{c}^{(k)}\right)$. Essentially, it is this comparison that gives a measure for the current models prediction capability. If the trial step $\boldsymbol{s}^{(k)}$ yields to a satisfactory decrease in the original objective functional in comparison to the one obtained by the model function, the iteration is successful, the trial step $\boldsymbol{s}^{(k)}$ is accepted and the model $m^{(k)}$ is updated i.e. a new POD ROM is derived that incorporates the flow dynamics as altered by the new control $\boldsymbol{c}^{(k+1)}$. Furthermore, if the achieved decrease in $f$ indicates a good behavior of the model $m^{(k)}$, the trust-region radius $\Delta^{(k)}$ can be increased. Now, if there is a limited predicted decrease compared to the actual decrease, we have the possibility to decrease slightly the value of the trust-region radius. For unsuccessful iterations, the trial step $\boldsymbol{s}^{(k)}$ is not accepted, the trust-region radius $\Delta^{(k)}$ is decreased and the trust-region subproblem (8) is solved again within a smaller trust-region. With the contraction of the trust-region it is more likely to have a good approximation to the true objective functional with the POD ROM. The corresponding TRPOD algorithm is schematically described in Fig. 3 and given in Appendix A. The proofs of global convergence of this algorithm are detailed in [50]. The main results can be found in [56].

\section{A robust POD-based estimator for drag function}

The objective of this study is the minimization of the mean time drag coefficient of the circular cylinder i.e. of the cost function $\mathcal{J}(\boldsymbol{U})$ given by (3). Here, $\boldsymbol{U}$ is solution of the Navier-Stokes equations (1). However, the value of the cost function $\mathcal{J}$ can also be evaluated from the state variables $\widehat{\boldsymbol{U}}$ rebuilt after integration of a POD based control model.

Consequently, while the real objective function writes

$$
\mathcal{J}(\boldsymbol{U})=\frac{1}{T} \int_{0}^{T} \mathcal{C}_{\mathcal{D}}(\boldsymbol{U}) \mathrm{d} t
$$

the model function is

$$
\mathcal{J}(\widehat{\boldsymbol{U}})=\frac{1}{T} \int_{0}^{T} \mathcal{C}_{\mathcal{D}}(\widehat{\boldsymbol{U}}) \mathrm{d} t
$$

Clearly, the pressure field appears in (10). However, for a Reynolds number roughly equal to 200 , it is well-known $[20,56]$ that the pressure term contributes 


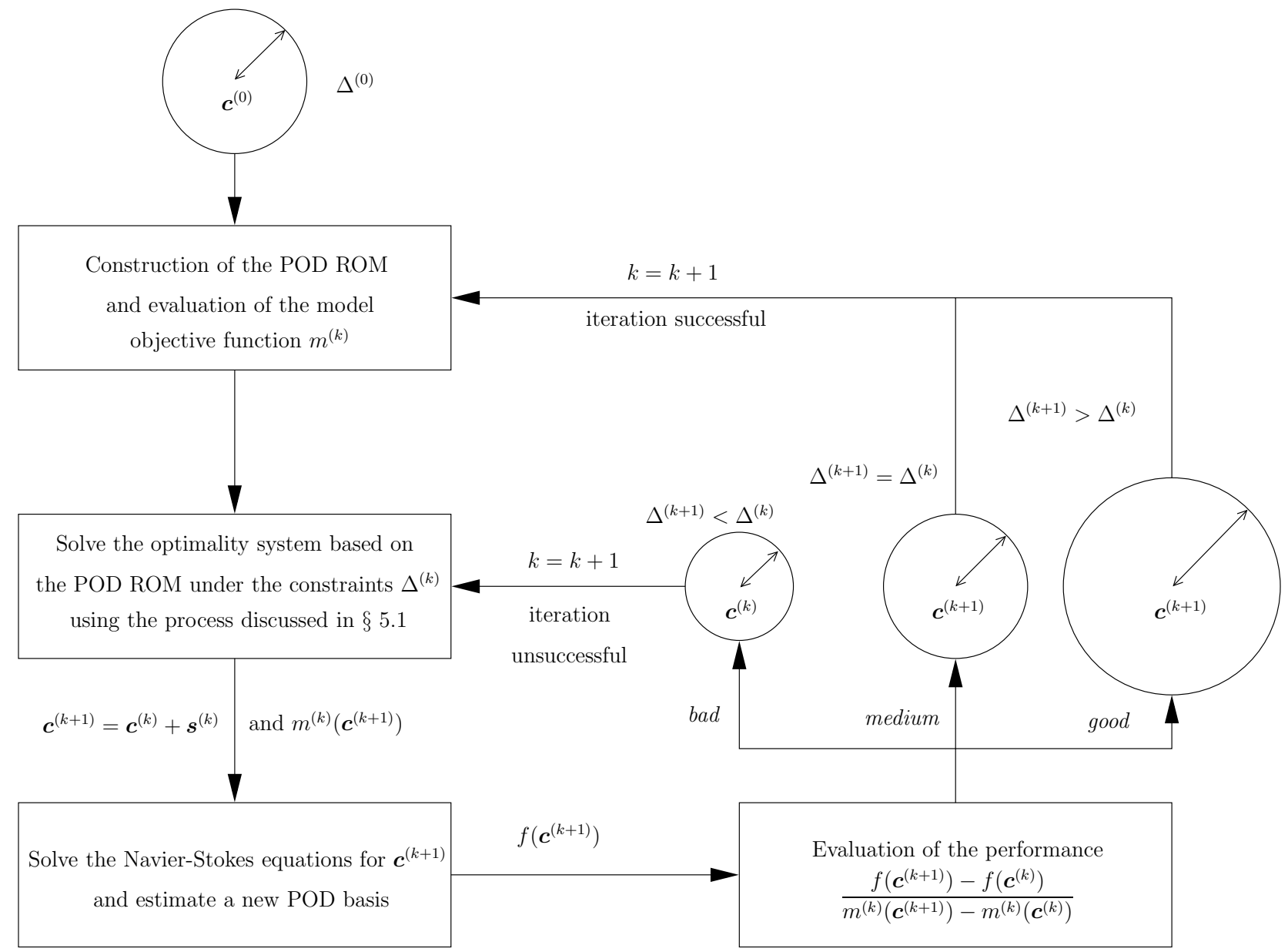

Fig. 3. Schematic representation of the TRPOD algorithm.

to approximately $80 \%$ of the total drag coefficient. So that the model function cost represents accurately the real function cost, it is thus necessary to include the pressure field in the POD model. This is the aim of the following section.

\subsection{POD reconstruction of the pressure field}

\subsubsection{Determination of a pressure POD basis}

In most of the POD applications, only the velocity field is decomposed. For experimental work, that can be quite simply explained by the fact that in the majority of the cases the pressure data is unavailable. The contribution of the pressure term is then neglected in the POD ROM. In many closed flows, it can be demonstrated rigorously that this contribution is exactly zero. For convectively unstable shear layers, as the mixing layer or the wake flow, it was proved in [57] that neglecting the pressure term may lead to large amplitude 
errors in the Galerkin model hence the need for introducing a pressure term representation $[57,58]$. For our application, pressure plays an important role and must be incorporated into the POD formulation. One way is to derive the pressure from the velocity field by solving a Poisson equation in the lowdimensional POD subspace. In this respect, the first analytical pressure term representation for open flows has been performed for POD Galerkin models based on the Navier-Stokes equations in primitive variables by [57]. This method seems natural for an incompressible flow. It is in addition the only one that can be used when no pressure field is available. However, solving a Poisson equation in the POD subspace requires the development of a specific Poisson solver. Moreover, this formulation of the problem does not constitute per se a reduced-order model of dynamics. A second option is to consider the state vector $\boldsymbol{U}$ as input-data for applying the POD technique. Here, since the data are issued from numerical simulations [48, for a justification], the snapshot POD introduced by [8] is adopted. Finally, after application of the POD, the field $\boldsymbol{U}$ can be expanded with arbitrary accuracy as

$$
\boldsymbol{U}(\boldsymbol{x}, t) \simeq \widehat{\boldsymbol{U}}^{\left[m, c, 1, \cdots, N_{\mathrm{POD}}\right]}(\boldsymbol{x}, t)=\boldsymbol{U}_{m}(\boldsymbol{x})+\gamma(\boldsymbol{c}, t) \boldsymbol{U}_{c}(\boldsymbol{x})+\sum_{i=1}^{N_{\mathrm{POD}}} a_{i}(t) \boldsymbol{\phi}_{i}(\boldsymbol{x})
$$

where $N_{\text {POD }}$ is equal to the number of flow realizations contained in the input ensemble used to determine the POD modes. For later reference, the estimation $\widehat{\boldsymbol{U}}^{\left[m, c, 1, \cdots, N_{\mathrm{POD}}\right]}$ of $\boldsymbol{U}$ is introduced, where the brackets contain the indices of all employed modes. In (11), the mean field $\boldsymbol{U}_{m}$, the actuation mode $\boldsymbol{U}_{c}$ and the POD basis functions $\left\{\phi_{i}=\left(\phi_{i}^{u}, \phi_{i}^{v}, \phi_{i}^{p}\right)^{T}\right\}_{i=1}^{N_{\mathrm{POD}}}$ are computed using the following algorithm:

(1) Use the control function method introduced in the POD ROM context by [16] to determine a reference flow field $\boldsymbol{U}_{c}(\boldsymbol{x})=\left(u_{c}(\boldsymbol{x}), v_{c}(\boldsymbol{x}), p_{c}(\boldsymbol{x})\right)^{T}$. Here $^{4}, \boldsymbol{U}_{c}$ is generated as the solution of the Navier-Stokes equations for a unit control $(\gamma=1)$ and homogeneous boundary conditions for the

${ }_{4}$ Recently, a general method was proposed in [59] to determine the actuation mode $\boldsymbol{U}_{c}$. This approach named control input separation consists in seeking the actuation mode $\boldsymbol{U}_{c}^{*}$ which is solution of the following optimization problem:

$$
\min _{\boldsymbol{U}_{c} \in \mathbb{H}} \sum_{i=1}^{N_{t}}\left\|\boldsymbol{U}_{\text {snap }}\left(\boldsymbol{x}, t_{i}\right)-P_{\mathcal{S}} \boldsymbol{U}_{\text {snap }}\left(\boldsymbol{x}, t_{i}\right)-\gamma\left(t_{i}\right) \boldsymbol{U}_{c}(\boldsymbol{x})\right\|_{\Omega}^{2}
$$

where $\mathbb{H}$ is a real Hilbert space and where $P_{\mathcal{S}}$ denotes projection onto $\mathcal{S}$, the space spanned by the POD eigenfunctions of the uncontrolled configuration $(\gamma=0)$. By definition, the choice $\boldsymbol{U}_{c}=\boldsymbol{U}_{c}^{*}$ is optimal, in the sense that the energy not captured by the expansion (11) achieves its minimum for $\boldsymbol{U}_{c}=\boldsymbol{U}_{c}^{*}$. In addition, it was shown in [59] that the actuation mode is not significantly dependent on the specific excitation $\gamma$ used to determine it. 
uncontrolled boundaries (see Fig. $6(\mathrm{k})$ for a streamlines representation of $\left.\boldsymbol{u}_{c}\right)$.

(2) Compute the mean flow $\boldsymbol{U}_{m}(\boldsymbol{x})$ as the ensemble average of the modified snapshots set $\left\{\boldsymbol{U}\left(\boldsymbol{x}, t_{i}\right)-\gamma\left(\boldsymbol{c}, t_{i}\right) \boldsymbol{U}_{c}(\boldsymbol{x})\right\}_{i=1}^{N_{t}}$ :

$$
\boldsymbol{U}_{m}(\boldsymbol{x})=\frac{1}{N_{t}} \sum_{i=1}^{N_{t}}\left\{\boldsymbol{U}\left(\boldsymbol{x}, t_{i}\right)-\gamma\left(\boldsymbol{c}, t_{i}\right) \boldsymbol{U}_{c}(\boldsymbol{x})\right\}
$$

where $\boldsymbol{U}\left(\boldsymbol{x}, t_{i}\right)$ correspond to $N_{t}$ flow realizations, taken at time instants $t_{i} \in[0, T], i=1, \cdots, N_{t}$. In the case of incomplete or noisy data sets (obstructed view of PIV measurements for instance), we need to mention that interpolation methods (Kriging or "gappy" POD) can be used to reconstruct unsteady flow fields $[60,61]$.

(3) Define the fluctuation fields $\boldsymbol{U}_{\text {snap }}$ :

$$
\boldsymbol{U}_{\text {snap }}\left(\boldsymbol{x}, t_{i}\right)=\boldsymbol{U}\left(\boldsymbol{x}, t_{i}\right)-\gamma\left(\boldsymbol{c}, t_{i}\right) \boldsymbol{U}_{c}(\boldsymbol{x})-\boldsymbol{U}_{m}(\boldsymbol{x}) .
$$

(4) Build the temporal correlation matrix $C$ of components $C_{i j}$ defined as:

$$
C_{i j}=\left(\boldsymbol{U}_{\text {snap }}\left(\boldsymbol{x}, t_{i}\right), \boldsymbol{U}_{\text {snap }}\left(\boldsymbol{x}, t_{j}\right)\right)_{\Omega}=\int_{\Omega} \boldsymbol{U}_{\text {snap }}\left(\boldsymbol{x}, t_{i}\right) \cdot \boldsymbol{U}_{\text {snap }}\left(\boldsymbol{x}, t_{j}\right) \mathrm{d} \boldsymbol{x} .
$$

(5) Compute the eigenvalues $\lambda_{1}, \cdots, \lambda_{N_{t}}$ and the temporal eigenvectors $\boldsymbol{\Psi}_{1}, \cdots, \boldsymbol{\Psi}_{N_{t}}$ of $C$ where $\boldsymbol{\Psi}_{j}=\left(\Psi_{j}\left(t_{1}\right), \Psi_{j}\left(t_{2}\right), \cdots, \Psi_{j}\left(t_{N_{t}}\right)\right)^{T}$.

(6) Compute spatial modes $\boldsymbol{\varphi}_{i}$ by linear combination of the temporal eigenvectors $\boldsymbol{\Psi}_{i}$ and the snapshots $\boldsymbol{U}_{\text {snap }}$ :

$$
\boldsymbol{\varphi}_{i}(\boldsymbol{x})=\sum_{j=1}^{N_{t}} \Psi_{i}\left(t_{j}\right) \boldsymbol{U}_{\text {snap }}\left(\boldsymbol{x}, t_{j}\right)
$$

(7) Normalize the modes $\boldsymbol{\varphi}_{i}$ to determine the spatial POD basis functions $\phi_{i}=\left(\phi_{i}^{u}, \phi_{i}^{v}, \phi_{i}^{p}\right)^{T}$ :

$$
\phi_{i}=\frac{\varphi_{i}}{\left\|\varphi_{i}\right\|_{\Omega}}
$$

As illustration of this procedure, a POD basis including the pressure field was computed from numerical snapshots of the controlled wake flow $(\gamma(t)=$ $A \sin \left(2 \pi S t_{f} t\right)$ with $A=2$ and $\left.S t_{f}=0.5\right)$. For that, 360 snapshots taken uniformly over $T_{\text {snap }}=18$ were considered. It was shown that the first 14 POD modes are necessary to represent $99.9 \%$ of the Relative Information Content defined, in function of the number of POD modes considered in the summation say $M$, as $\operatorname{RIC}(M)=\sum_{i=1}^{M} \lambda_{i} / \sum_{i=1}^{N_{\mathrm{POD}}} \lambda_{i}$. The norm of the first six pressure modes are displayed in Fig 4 . Similar representations for the velocity POD modes can be found in [52]. 


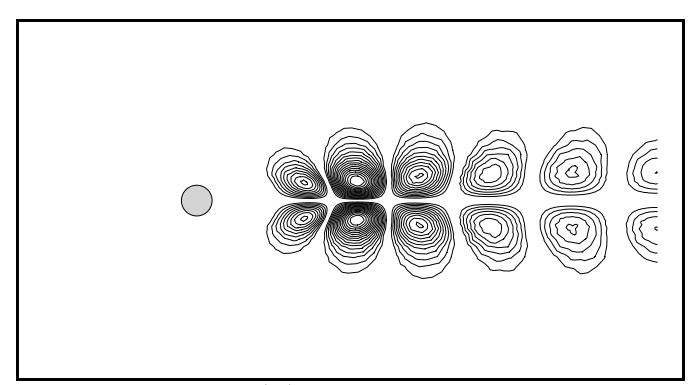

(a) mode 1 .

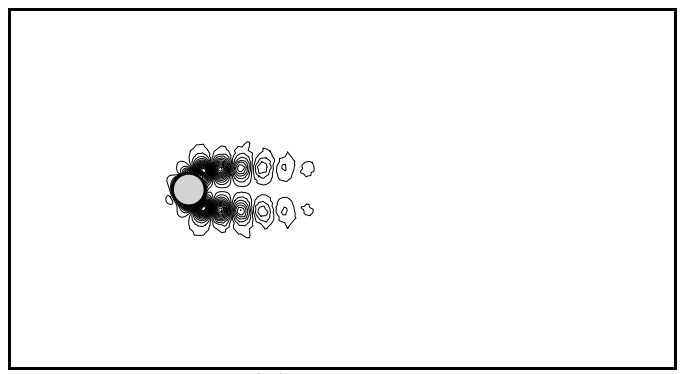

(c) mode 3 .

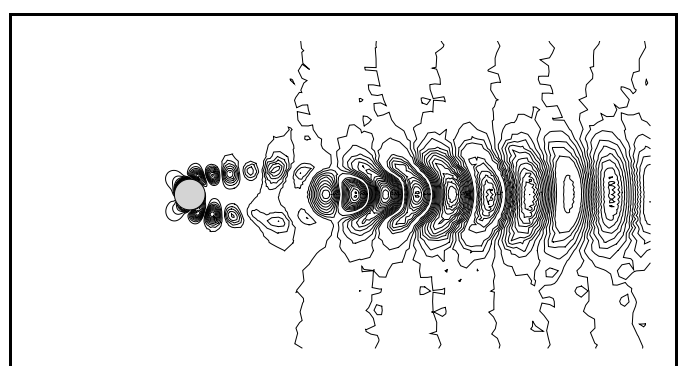

(e) mode 5 .

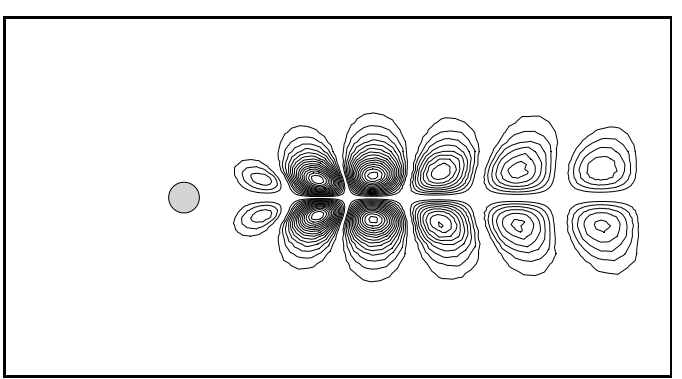

(b) mode 2 .

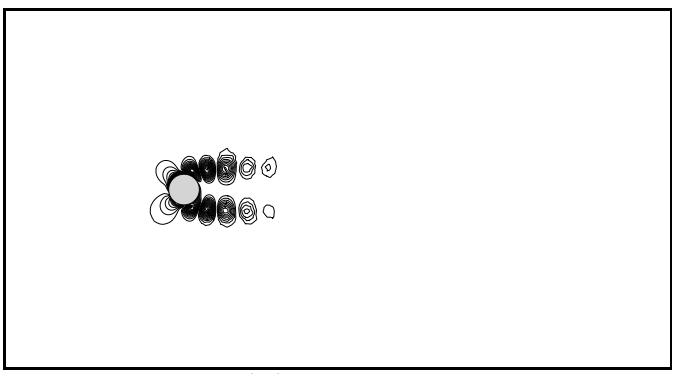

(d) mode 4 .

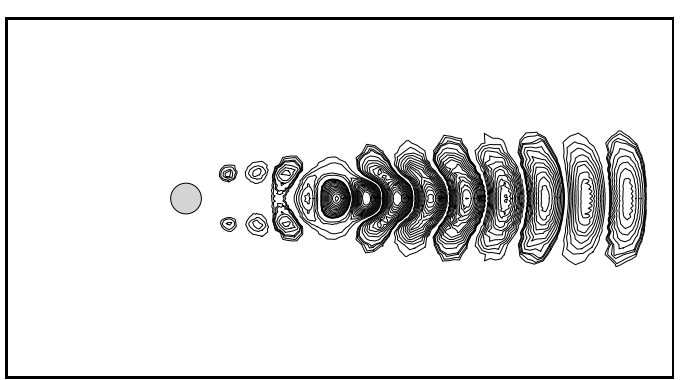

(f) mode 6 .

Fig. 4. Pressure POD basis functions for the controlled cylinder wake at $R e=200$. The first six POD modes $\phi_{n}^{p}$ are visualized by iso-contour lines of their norm $\left(\left\|\phi_{n}^{p}\right\|_{\Omega}\right)$ for $\gamma(t)=A \sin \left(2 \pi S t_{f} t\right)$ with $A=2$ and $S t_{f}=0.5$.

\subsubsection{POD ROM of the controlled cylinder wake}

The derivation of the POD ROM for the controlled cylinder wake is described in details in $\S I V . B$ of [15] in the case of eigenfunctions based only on velocity fields. As a matter of interest, it is shown that a Galerkin projection of the Navier-Stokes equations on the space spanned by the first $N_{\text {gal }}+1$ POD modes yields to:

$$
\begin{aligned}
\frac{\mathrm{d} a_{i}(t)}{\mathrm{d} t}= & \mathcal{A}_{i}+\sum_{j=0}^{N_{\text {gal }}} \mathcal{B}_{i j} a_{j}(t)+\sum_{j=0}^{N_{\text {gal }}} \sum_{k=0}^{N_{\text {gal }}} \mathcal{C}_{i j k} a_{j}(t) a_{k}(t) \\
& +\mathcal{D}_{i} \frac{\mathrm{d} \gamma}{\mathrm{d} t}+\left(\mathcal{E}_{i}+\sum_{j=0}^{N_{\text {gal }}} \mathcal{F}_{i j} a_{j}(t)\right) \gamma(\boldsymbol{c}, t)+\mathcal{G}_{i} \gamma^{2}(\boldsymbol{c}, t) \quad i=0, \cdots, N_{\text {gal }},
\end{aligned}
$$


with the following initial conditions:

$$
a_{i}(0)=\left(\boldsymbol{u}(\boldsymbol{x}, 0)-\boldsymbol{u}_{m}(\boldsymbol{x})-\gamma(\boldsymbol{c}, 0) \boldsymbol{u}_{c}(\boldsymbol{x}), \phi_{i}(\boldsymbol{x})\right)_{\Omega} \quad i=0, \cdots, N_{\text {gal }} .
$$

In (12), $N_{\text {gal }}$ is usually determined as the smallest number of POD modes necessary to represent $99.9 \%$ of the Relative Information Content and the mode $i=0$ correspond to the mean flow field $\boldsymbol{u}_{m}$. For an uncontrolled flow, this mode is typically not solved in the POD ROM [62, for instance] because its amplitude is supposed to be constant in time (equal to 1). Finally, the coefficients $\mathcal{A}_{i}, \mathcal{B}_{i j}, \mathcal{C}_{i j k}, \mathcal{D}_{i}, \mathcal{E}_{i}, \mathcal{F}_{i j}$ and $\mathcal{G}_{i}$ depend explicitly on $\boldsymbol{\phi}, \boldsymbol{u}_{m}$ and $\boldsymbol{u}_{c}$. Their expressions are given in [15].

Rigorously, the expression (12) cannot be used in our case since POD eigenfunctions now represent velocity and pressure fields. However, in this study, we will consider that this expression remains valid and determine the coefficients of the POD ROM with calibration methods based on optimization problems [63-65]. These calibration techniques are similar to those which had been used in [15] to represent accurately the controlled dynamics of reference with a POD ROM based on velocity only. The validity of this representation is now evaluated.

\subsubsection{Accuracy of the calibrated POD model}

The ability of the calibrated POD ROM to represent correctly the dynamics of the controlled flow can be assessed by the time evolution of the relative error based on $\boldsymbol{u}, E_{\boldsymbol{u}}$. This error measure is defined as:

$$
E_{\boldsymbol{u}}^{2}(t)=\frac{\left(\boldsymbol{u}-\widehat{\boldsymbol{u}}^{\left[m, c, 1, \cdots, N_{\text {gal }}\right]}, \boldsymbol{u}-\widehat{\boldsymbol{u}}^{\left[m, c, 1, \cdots, N_{\text {gal }}\right]}\right)_{\Omega}}{(\boldsymbol{u}, \boldsymbol{u})_{\Omega}},
$$

where $\widehat{\boldsymbol{u}}^{\left[m, c, 1, \cdots, N_{\text {gal }}\right]}$ corresponds to the approximation of the exact flow $\boldsymbol{u}$ when only the first $N_{\text {gal }}$ POD modes are retained in (11). A similar truncation error $E_{p}$ based on $p$ can be defined.

For a given control $\boldsymbol{c}$, the approximations $\widehat{\boldsymbol{u}}$ and $\widehat{p}$ can be evaluated either using the projection coefficients, or using the prediction coefficients obtained by numerical integration of the controlled POD ROM based on $\boldsymbol{U}$. Figure 5 represents a comparison of the corresponding errors for the projected modes (projection error) and the predicted modes (prediction error). As it could be expected from the optimality of the POD modes, the values of the projection error remain low throughout the time window, the amplitude of $E_{p}$ being even 


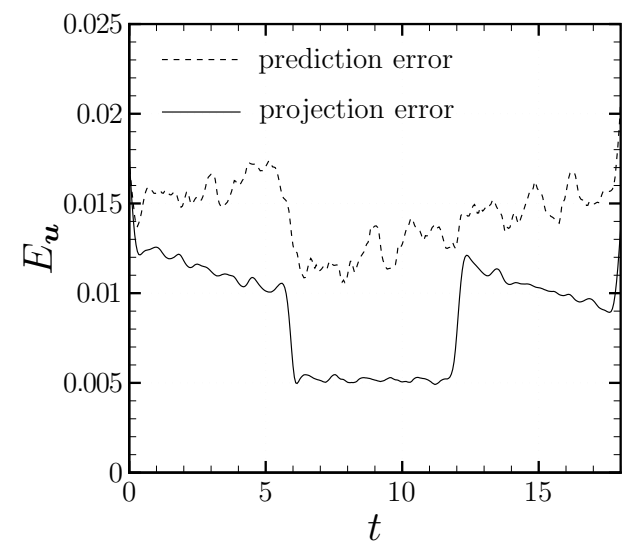

(a) Relative errors based on $\boldsymbol{u}$.

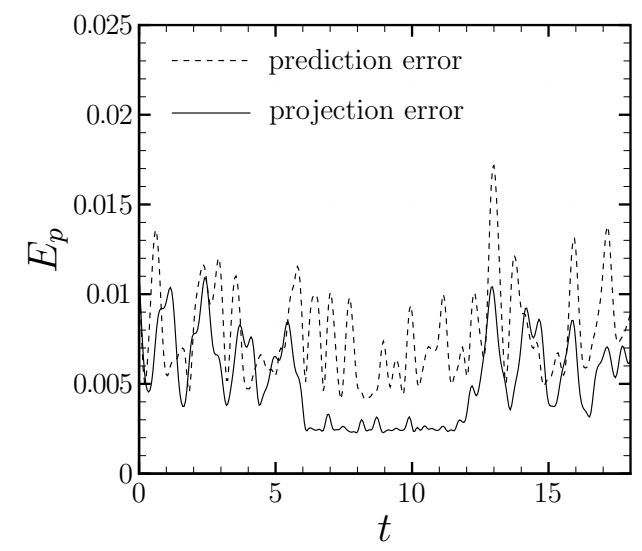

(b) Relative errors based on $p$.

Fig. 5. Comparison of the prediction and projection relative errors based on $\boldsymbol{u}$ and on $p$ for the controlled cylinder wake flow $\left(\gamma(t)=A \sin \left(2 \pi S t_{f} t\right)\right.$ with $A=2$ and $\left.S t_{f}=0.5\right)$. The approximations $\widehat{\boldsymbol{u}}$ and $\widehat{p}$ are evaluated for $N_{\text {gal }}=14$.

lower ${ }^{5}$ than that of $E_{\boldsymbol{u}}$. As for the prediction error, its values are slightly higher than those obtained for the projection error but no time amplification of the errors is observed. Consequently, one can consider that the calibrated POD ROM based on $\boldsymbol{U}$ represents accurately the temporal dynamics of the velocity and pressure fields, at least for values of the control $\boldsymbol{c}$ close to those used for the design. However, although the range of the POD ROM cannot be evaluated precisely, it is well-known that the performances of the model tend to deteriorate quickly with the change of the control parameters $[66,13]$. Recently, Noack et al. [14] reviewed the key enablers to the use of empirical Galerkin models for feedback flow control and suggested the introduction of non-equilibrium modes in the POD expansion as a way to enhance the range of validity of the controlled POD ROM.

\subsection{POD basis functions with non-equilibrium modes}

To describe two or more operating conditions in a single POD expansion, Noack et al. $[13,14]$ proposed to add special modes, called non-equilibrium modes, to the original POD basis functions. Essentially, these non-equilibrium modes will be, either particular modes not taken into account in the original model but known to play a major role in the description of the flow dynamics (stability eigenmodes for example), or translation modes (also called shift modes) that allow the description of the transition (natural or forced) from

\footnotetext{
5 This result should however be moderated because the error made on the representation of the velocity field $\boldsymbol{u}$ is the sum of the errors on the components $u$ and $v$.
} 
the uncontrolled configuration to the optimal controlled flow. Orthonormality of the POD basis functions is then enforced in the enlarged set of modes using a Gram-Schmidt procedure described in [13]. In that paper, Noack et al. demonstrated that the inclusion of a shift mode representing the mean field correction in an empirical Galerkin model of a wake flow significantly improves the resolution of the transient dynamics from the onset of vortex shedding to the periodic von Kármán vortex street. They also showed that the inclusion of stability eigenmodes further enhances the accuracy of fluctuation dynamics.

The velocity and pressure fields $\boldsymbol{U}$ can then be expanded as

$$
\begin{aligned}
\boldsymbol{U}(\boldsymbol{x}, t) & \simeq \widehat{\boldsymbol{U}}^{\left[c, 0, \cdots, N_{\text {gal }}^{r}+N_{\text {neq }}\right]}(\boldsymbol{x}, t) \\
& =\sum_{i=0}^{N_{\text {gal }}^{r}} a_{i}(t) \boldsymbol{\phi}_{i}(\boldsymbol{x})+\sum_{i=N_{\text {gal }}^{r}+1}^{N_{\text {gal }}^{r}+N_{\text {neq }}} a_{i}(t) \phi_{i}(\boldsymbol{x})+\gamma(\boldsymbol{c}, t) \boldsymbol{U}_{c}(\boldsymbol{x}) .
\end{aligned}
$$

Three different types of modes are employed in this expansion:

(1) POD modes: $\left(N_{\text {gal }}^{r}+1\right)$ POD modes are used to represent the dynamics of the reference operating condition. Here, the value of $N_{\text {gal }}^{r}$ is determined by using exactly the same energetic criterion that was discussed in section 4.1 .2 for $N_{\text {gal }}$.

(2) non-equilibrium modes: $N_{\text {neq }}$ non-equilibrium modes are added to describe new operating conditions. When non-equilibrium modes are introduced in the model to represent the dynamics of controlled configurations, the mean flow mode $(i=0)$ can have a transient state during which energy is exchanged with the non-equilibrium modes.

(3) actuation mode: $\boldsymbol{U}_{c}$ is determined by the control function method. This method is used to introduce explicitly the control in the model. The actuation mode corresponds to predetermined dynamics. It is thus not modified by the dynamical evolutions intervening during the control process.

A controlled POD ROM similar to that described in section 4.1 .2 can thus be derived. This model is different from the previous one essentially by the modes used in the Galerkin projection. After calibration, this model is that used in the remainder of the article to describe the controlled dynamics of the wake flow. To simplify, we will continue to refer to (12) for the system of equations of the controlled POD ROM.

As it was already discussed at length in the introduction, the principal difficulty in the use of a POD ROM to solve an optimization problem is that neither the optimal parameters, nor the optimal path in the control parameter space are known in advance. Consequently, if the dynamics $I$ corresponding to the uncontrolled flow is naturally known, it is impossible to know in advance 
what are the dynamics $I I, I I I, I V, \cdots$ which will be the most relevant to introduce in the model. Recent work [10] seems to demonstrate that centroidal Voronoi tessellations could be one method of intelligent sampling in parameter space. Here, a simpler method is adopted, the snapshots being taken randomly in the control parameter space. Hereafter, the following dynamics are considered:

- dynamics $I$ : controlled flow with $A=2$ and $S t=0.5$,

- dynamics $I I$ : controlled flow with $A=4$ and $S t=0.1$,

- dynamics $I I I$ : natural flow $A=0$,

- dynamics $I V$ : unstable steady basic flow. It was argued in [30] that this configuration corresponds to the lowest mean drag reduction that can be achieved under rotary control of the cylinder.

In addition, since the objective is the mean drag reduction of the wake flow, the non-equilibrium modes will correspond only to averaged flows. The main POD and non-equilibrium modes used to derive the POD ROM (12) are represented on Fig. 6. Finally, after integration in time, the solutions $\left\{a_{i}\right\}_{i=0}^{N_{\text {gal }}^{r}+N_{\text {neq }}}$ of the model (12) can be used to approximate the drag coefficient.

\subsection{Construction of the surrogate drag function}

If the drag operator introduced in (2) is applied to expansion (13), a model function of the drag coefficient is obtained:

$$
{\widehat{C_{D}}}^{\left[c, 0, \cdots, N_{\text {gal }}^{r}+N_{\text {neq }}\right]}(t)=\mathcal{C}_{\mathcal{D}}\left(\widehat{\boldsymbol{U}}^{\left[c, 0, \cdots, N_{\text {gal }}^{r}+N_{\text {neq }}\right]}\right)=\gamma(\boldsymbol{c}, t) \mathcal{C}_{\mathcal{D}}\left(\boldsymbol{U}_{c}\right)+\sum_{i=0}^{N_{\text {gal }}^{r}+N_{\text {neq }}} a_{i}(t) \mathcal{C}_{\mathcal{D}}\left(\phi_{i}\right)
$$

Since the field $\boldsymbol{U}_{c}$ is predetermined and have circular symmetry (see Fig. 6(k) for the actuation mode $\boldsymbol{u}_{c}$ for example), it does not contribute to the drag coefficient and thus $\mathcal{C}_{\mathcal{D}}\left(\boldsymbol{U}_{c}\right) \equiv 0$. Put $K_{i}=\mathcal{C}_{\mathcal{D}}\left(\phi_{i}\right)$, the model function becomes:

$$
{\widehat{C_{D}}}^{\left[c, 0, \cdots, N_{\text {gal }}^{r}+N_{\mathrm{neq}}\right]}(t)=\sum_{i=0}^{N_{\mathrm{gal}}^{r}} a_{i}(t) K_{i}+\sum_{i=N_{\mathrm{gal}}^{r}+1}^{N_{\mathrm{gal}}^{r}+N_{\mathrm{neq}}} a_{i}(t) K_{i}
$$

To highlight in this expression the relative contributions of the weakly unsteady terms and the strongly unsteady fluctuations, this model function can 


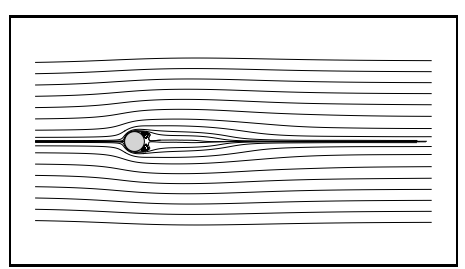

(a) Mean controlled flow $I$.

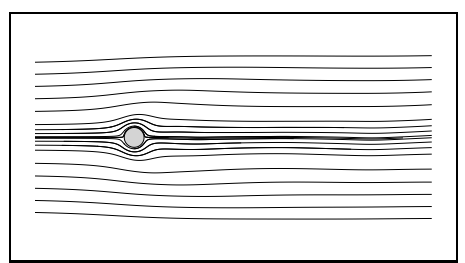

(d) Mean controlled flow II.

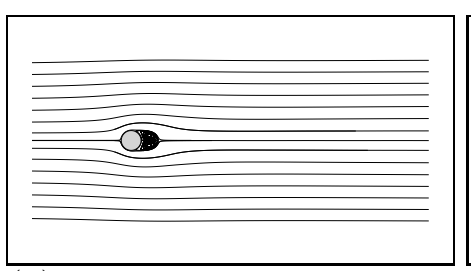

(g) Mean natural flow III.

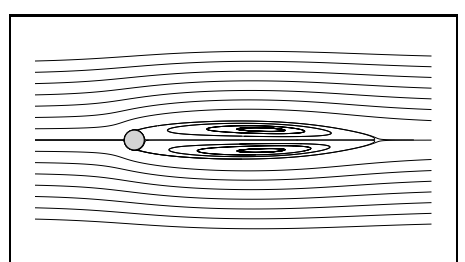

(j) Steady basic flow $I V$.

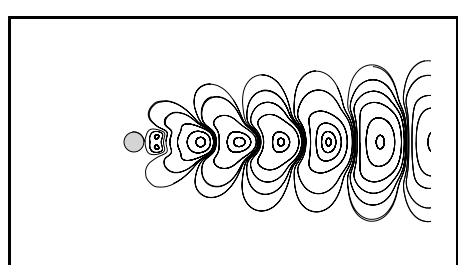

(b) 1st POD mode of $I$.

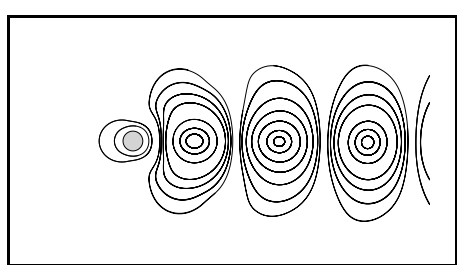

(e) 1st POD mode of $I I$.

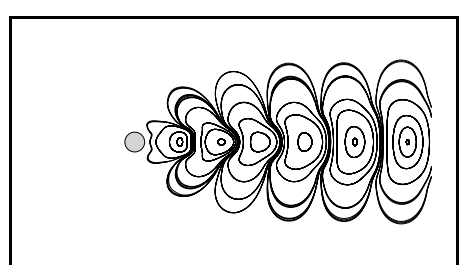

(c) 2nd POD mode of $I$.

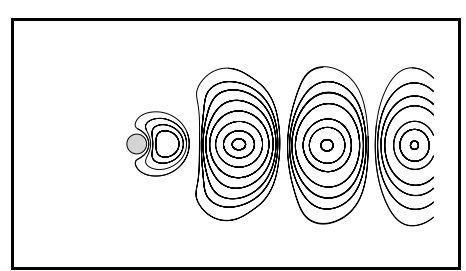

(f) 2nd POD mode of $I I$.
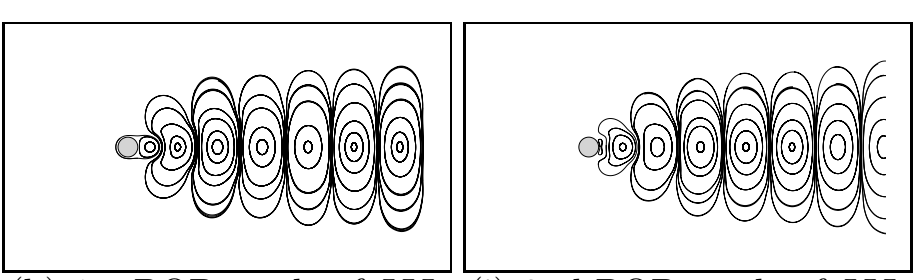

(h) 1st POD mode of $I I I$. (i) 2nd POD mode of $I I I$.

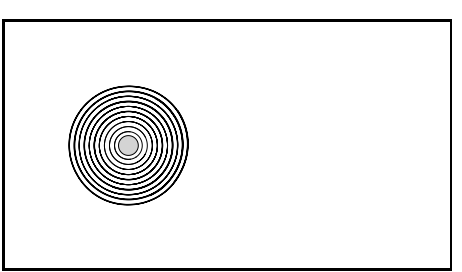

(k) Actuation mode $\boldsymbol{u}_{c}$.

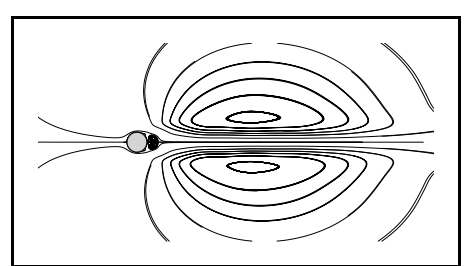

( $\ell$ ) Shift mode from $I I I$ to $I V$.
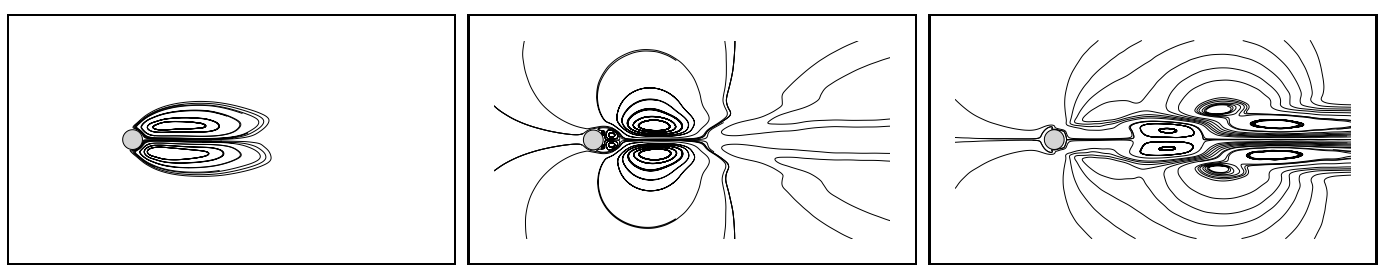

(m) Shift mode from $I$ to (n) Shift mode from $I$ to (o) Shift mode from $I$ to II. III. $I V$.

Fig. 6. Modes considered in the reduced-order control model (12) of the cylinder wake at $R e=200$. In all sub-figures, the flow is visualized with streamlines. Note that subfigure 6(d) looks like a potential solution and that subfigure $6(\mathrm{k})$ approximates a potential vortex.

be rewritten:

$$
{\widehat{C_{D}}}^{\left[c, 0, \cdots, N_{\text {gal }}^{r}+N_{\text {neq }}\right]}(t)=\underbrace{a_{0}(t) K_{0}+\sum_{i=N_{\text {gal }}^{r}+1}^{N_{\text {gal }}^{r}+N_{\text {neq }}} a_{i}(t) K_{i}}_{\text {evolution of the mean drag }}+\underbrace{\sum_{i=1}^{N_{\text {gal }}^{r}} a_{i}(t) K_{i}}_{\text {fluctuations } C_{D}^{\prime}}
$$




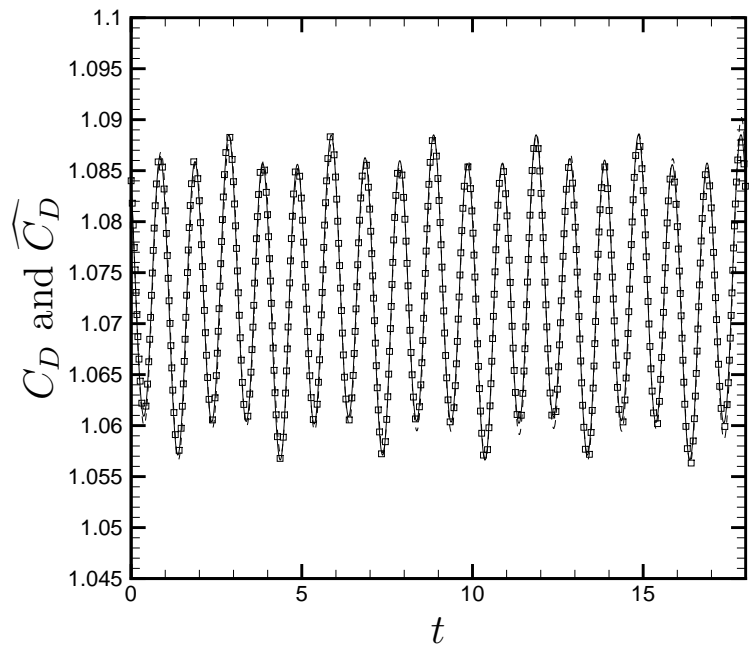

Fig. 7. Comparison of the real drag coefficient $C_{D}$ (symbols) and model function (lines) of the drag coefficient for the controlled cylinder wake flow $\left(\gamma(t)=A \sin \left(2 \pi S t_{f} t\right)\right.$ with $A=2$ and $\left.S t_{f}=0.5\right)$.

Finally, since the average of the fluctuations estimated over a finite time horizon $T$ equal to a few periods of vortex shedding is approximately null, the model objective function could be written as:

$$
\widehat{\mathcal{J}}=\left\langle{\widehat{C_{D}}}^{\left[c, 0, \cdots, N_{\mathrm{gal}}^{r}+N_{\mathrm{neq}}\right]}(t)\right\rangle_{T}=\frac{1}{T} \int_{0}^{T}\left(a_{0}(t) K_{0}+\sum_{i=N_{\mathrm{gal}}^{r}+1}^{N_{\mathrm{gal}}^{r}+N_{\mathrm{neq}}} a_{i}(t) K_{i}\right) \mathrm{d} t .
$$

The robustness of these model functions to the variations of the flow control parameters is measured by the capacity which has the POD ROM (12) to represent the variations of the real mean drag coefficient when the control law $\gamma$ used for the numerical integration of the system varies. Indeed, even if the POD basis functions $\phi_{i}$ employed for the Galerkin projection correspond to reference control parameters, the coefficients $a_{i}$ depend implicitly on the specific control law used to solve the system (12). For example, if this system is solved with a control law $\gamma$ identical to that used to derive the model then the coefficient $a_{0}$ is approximately equal to a constant and the terms $\left\{a_{i}\right\}_{i=N_{\text {gal }}^{r}+1}^{N_{\text {gal }}^{r}+N_{\text {neq }}}$ are all identically null. The mean field then tends towards $a_{0} \phi_{0}$ and the value of the objective function converges towards $a_{0} K_{0}$. On the other hand, if the system (12) is solved with a value of $\gamma$ different from that used to derive the model then it is possible that the mode $a_{0}$ interacts with the non-equilibrium modes $\left\{a_{i}\right\}_{i=N_{\text {gal }}^{r}+1}^{N_{\text {al }}^{r}+N_{\text {neq }}}$ leading to a variation of the mean drag coefficient.

The model function of the drag coefficient is validated once again for the controlled wake flow characterized by $A=2$ and $S t_{f}=0.5$. Figure 7 dis- 


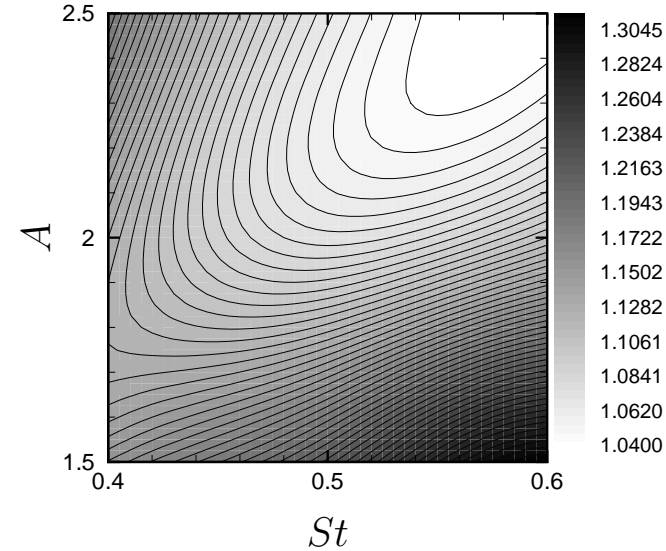

(a) Real objective function $\mathcal{J}$.

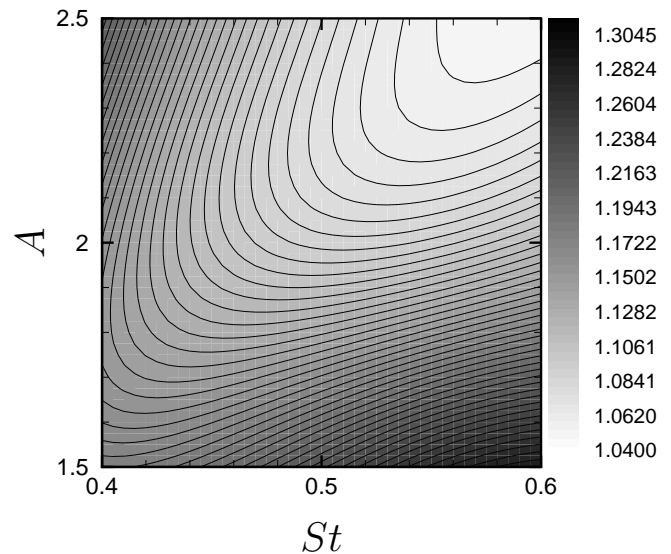

(b) Model objective function $\widehat{\mathcal{J}}$.

Fig. 8. Iso-values of the real and model objective functions associated to the mean drag coefficient. The same contour levels are used in the two figures.

plays a comparison of the real drag coefficient obtained numerically for the Navier-Stokes equations and the model function of the drag coefficient (15) determined after time integration of the system (12). At the design parameters, excellent qualitative agreements are obtained not only for the mean drag coefficient but also for the amplitudes and the characteristic frequencies of the oscillations. The next stage consists in checking if the model objective function (16) is suitable to represent the behavior of the real objective function (9) for control parameters $A$ and $S t_{f}$ close to those corresponding to the reference flow. The dynamical system (12) is then integrated in time for various values of the control parameters located in a domain $\mathcal{D}$ centered on the reference parameters $A=2$ and $S t_{f}=0.5$. For $\mathcal{D}=\{1.5 \leq A \leq 2.5 ; 0.4 \leq S t \leq 0.6\}$, the iso-values of the real and model objective functions, respectively $\mathcal{J}$ and $\widehat{\mathcal{J}}$, are compared in Fig. 8. The values and the variations of these two functions are similar on the domain $\mathcal{D}$ (the maximum value of the relative error is equal to $3.9 \%$ ), thus validating the choice of the model function (16). Thereafter, this model function will thus be used to determine, using the TRPOD algorithm described in section 3, the control law that minimizes the mean drag coefficient. Finally, note that the use of POD expansions without the addition of non-equilibrium modes or a too large domain $\mathcal{D}$ can lead to erroneous results [56].

\section{Drag minimization of the cylinder wake flow by POD-based adaptive controllers}

One major difficulty with surrogate optimization based on POD ROM is that models derived for a specific controlled flow are possibly unreliable to represent 
the flow altered by another control. We then propose in this section different reduced-order adaptive procedures that improve the models by successively updating the snapshot data. Essentially, these optimization algorithms differ by the criterion which is used to decide whether or not a reduced-order model has to be adapted to a new flow configuration. In section 5.1, the optimal control approach used to solve the constrained optimization subproblem (8) is first described. Then, the numerical results obtained with two different strategies of adaptive controllers are presented. In section 5.2.1, a suboptimal controller corresponding to a simplified version of the TRPOD algorithm is considered. Then, the optimal solutions determined with the TRPOD algorithm are presented ( $(5.2 .2)$. Finally, the energetic efficiency of our approach is discussed and the cost reduction factors are estimated $(\S 5.2 .3)$.

\subsection{Optimal control approach}

The convergence behavior of trust-region methods for general model functions with inexact gradient information is usually based on a sufficient decrease condition of the objective function [54, for example]. In his original work, Fahl [50] extended these classical results and demonstrated that the exact solution of the subproblem (8) is not necessary to prove global convergence of the TRPOD algorithm. Here, because of the low computational costs of solving the POD reduced-order models, an exact optimal solution of the subproblem (8) is directly sought. For that, this subproblem is first reformulated as a constrained optimization problem which is then solved by the Lagrange multipliers method as described in [2].

Moving all the terms in the left hand side, the state equations (12) are written more simply as ${ }^{6}$

$$
\mathcal{N}_{i}(\boldsymbol{a}, \boldsymbol{c})=0 \quad i=0, \cdots, N_{\text {gal }}^{r}+N_{\text {neq }}
$$

where $\boldsymbol{a}$ is the vector containing the time-dependent expansion coefficients $\left\{a_{i}\right\}_{i=0}^{N_{\text {gal }}^{r}+N_{\text {neq }}}$ and $\boldsymbol{c}$ is the control vector whose components are the amplitude $A$ and the Strouhal number $S t_{f}$ which define the sinusoidal control law $\gamma(t)$.

Thereafter, to simplify the various writings, the fluctuation term $C_{D}^{\prime}(t)=$ $\sum_{i=1}^{N_{\text {gal }}^{r}} a_{i}(t) K_{i}$ of the drag coefficient is included in (16). Therefore, the model objective function $\widehat{\mathcal{J}}$ is expressed as

$$
\widehat{\mathcal{J}}(\boldsymbol{a})=\frac{1}{T} \int_{0}^{T} J(\boldsymbol{a}) \mathrm{d} t
$$

\footnotetext{
$\overline{6}$ In order to simplify the expressions of the optimality system, we will not specify in the notations of this section that the state equations depend on the iteration number $k$ of the TRPOD algorihtm.
} 
where $J(\boldsymbol{a})=\sum_{i=0}^{N_{\text {gal }}^{r}+N_{\text {neq }}} a_{i}(t) K_{i}$. In this expression, the state variables $\boldsymbol{a}$ depend implicitly of the control $\boldsymbol{c}$ used to integrate the state equations (17).

However, since we are interested by the exact minimization of the objective function $\widehat{\mathcal{J}}$ in the trust-region characterized by its radius $\Delta$, it is useless to introduce a penalization term expressing the cost of the control as that is usually done. In the same way, the objective function $\widehat{\mathcal{J}}$ being sufficiently regular (see Fig. 2), the minimization of $\widehat{\mathcal{J}}$ in the trust-region is well-posed and it is not more necessary to introduce a Tikhonov-type regularization term as in [39].

Finally, the constrained optimization problem

$$
\min _{\boldsymbol{c}} \widehat{\mathcal{J}}(\boldsymbol{a}) \quad \text { subject to } \quad \mathcal{N}_{i}(\boldsymbol{a}, \boldsymbol{c})=0 \quad i=0, \cdots, N_{\text {gal }}^{r}+N_{\text {neq }},
$$

is transformed to an unconstrained optimization problem by defining the Lagrangian functional

$$
\mathcal{L}(\boldsymbol{a}, \boldsymbol{c}, \boldsymbol{\xi})=\frac{1}{T} \int_{0}^{T}\left(J(\boldsymbol{a})-\sum_{i=0}^{N_{\mathrm{gal}}^{r}+N_{\mathrm{neq}}} \xi_{i} \mathcal{N}_{i}(\boldsymbol{a}, \boldsymbol{c})\right) \mathrm{d} t
$$

where $\boldsymbol{\xi}$ are Lagrange multipliers (also known as adjoint state variables) that enforce the state equations (17). The optimality system can be derived by taking variations of the Lagrangian with respect to the adjoint, state and control variables.

Setting the first variation of the Lagrangian with respect to the Lagrange multipliers $\boldsymbol{\xi}$ equal to zero and arguing that the variation of $\boldsymbol{\xi}$ is arbitrary in $[0, T]$, simply recovers the state equations (17).

Setting the first variation of $\mathcal{L}$ with respect to the state variables $\boldsymbol{a}$ to zero and arguing that the variation of $\boldsymbol{a}$ is arbitrary in $[0, T]$, and at $t=T$, yields the adjoint equations:

$$
\begin{aligned}
\frac{\mathrm{d} \xi_{i}(t)}{\mathrm{d} t}= & -\sum_{j=0}^{N_{\text {gal }}^{r}+N_{\text {neq }}}\left(\mathcal{B}_{j i}+\gamma(\boldsymbol{c}, t) \mathcal{F}_{j i}+\sum_{k=0}^{N_{\text {gal }}^{r}+N_{\text {neq }}}\left(\mathcal{C}_{j i k}+\mathcal{C}_{j k i}\right) a_{k}(t)\right) \xi_{j}(t) \\
& -\frac{1}{T} K_{i},
\end{aligned}
$$

and the terminal conditions:

$$
\xi_{i}(T)=0
$$

Note that the adjoint system (21) is posed backward in time, i.e. terminal conditions are given at $t=T$ instead of initial conditions. 
Defining $\mathcal{L}_{i}=-\frac{\mathrm{d} \xi_{i}}{\mathrm{~d} t} \mathcal{D}_{i}+\xi_{i}\left(\mathcal{E}_{i}+\sum_{j=0}^{N_{\text {gal }}^{r}+N_{\text {neq }}} \mathcal{F}_{i j} a_{j}+2 \gamma(\boldsymbol{c}, t) \mathcal{G}_{i}\right)$ and taking the derivative of the Lagrangian with respect to the control variables $\boldsymbol{c}$ yields the following vector equation

$$
\nabla_{c} \widehat{\mathcal{J}}=\frac{1}{T} \int_{0}^{T}\left(\sum_{i=0}^{N_{\text {gal }}^{r}+N_{\text {neq }}} \mathcal{L}_{i}\right) \nabla_{c} \gamma \mathrm{d} t
$$

which can be projected onto the two control directions $A$ and $S t_{f}$ to get:

$$
\delta \widehat{\mathcal{J}}_{A}=\frac{1}{T} \int_{0}^{T}\left(\sum_{i=0}^{N_{\text {gal }}^{r}+N_{\text {neq }}} \mathcal{L}_{i}\right) \sin \left(2 \pi S t_{f} t\right) \mathrm{d} t,
$$

and

$$
\delta \widehat{\mathcal{J}}_{S t_{f}}=\frac{1}{T} \int_{0}^{T} 2 \pi A t\left(\sum_{i=0}^{N_{\mathrm{gal}}^{r}+N_{\mathrm{neq}}} \mathcal{L}_{i}\right) \cos \left(2 \pi S t_{f} t\right) \mathrm{d} t
$$

These equations, also known as optimality conditions, are only equal to zero at the minimum of the objective function.

The optimality system formed by the state equations (12), the adjoint equations (21) and the optimality conditions (23a) and (23b) is solved with an iterative procedure similar to that used in [15]. In short, a direction of descent is determined by the Fletcher-Reeves version of the Conjugate Gradient Method and the line search strategy is ensured at each iteration by the backtracking Armijo method. More details can be found in [52]. The iterative method is stopped when two following values of the functional $\widehat{\mathcal{J}}$ are sufficiently close i.e. when $|\Delta \widehat{\mathcal{J}}(\boldsymbol{a})|=\left|\widehat{\mathcal{J}}^{(n+1)}(\boldsymbol{a})-\widehat{\mathcal{J}}^{(n)}(\boldsymbol{a})\right|<10^{-5}$ where $n$ represents the iteration number.

\subsection{Numerical results of two POD-based adaptive controllers}

In the original version of the TRPOD approach (see algorithm A for a thorough description and Fig. 3 for a schematic representation), the radius $\Delta^{(k)}$ of the trust-region is modified, if necessary, at each iteration $k$ by comparing the actual reduction of the true objective function $\mathcal{J}(\boldsymbol{U}(\boldsymbol{c}))$ to the predicted reduction obtained with the model function $m=\widehat{\mathcal{J}}(\widehat{\boldsymbol{U}}(\boldsymbol{c}))$. An elementary modification of this algorithm consists in considering the radius of the trust-region constant throughout the optimization process $\left(\Delta^{(k+1)}=\Delta^{(k)}\right)$ and equal to a finite or infinite given value. The results obtained for this simplified version of the TRPOD algorithm are presented in section 5.2.1. Those obtained by the TRPOD algorithm A are presented in section 5.2.2. In both cases, the 
stopping criterion of the adaptive procedure is $\left|\widehat{\mathcal{J}}^{(k)}-\widehat{\mathcal{J}}^{(k+1)}\right|<\varepsilon$ where the tolerance $\varepsilon$ is arbitrarily taken equal to $10^{-5}$.

As it was discussed for example in [36], a possible drawback of solving a minimization problem with a gradient-based optimization approach is that the algorithm may converge to the global minimum or to some other local minimum of the cost function depending on the relative position of the starting point to the minima. To alleviate this difficulty and evaluate the robustness of the two adaptive controllers, the optimization process will be initialized starting from several different control $\boldsymbol{c}^{(0)}$ chosen at random in the control parameter space retained for the open-loop control procedure (see Fig. 2). Hereafter, four different initial values are employed: $\boldsymbol{c}^{(0)}=(1.0,0.2)^{T}, \boldsymbol{c}^{(0)}=$ $(1.0,1.0)^{T}, \boldsymbol{c}^{(0)}=(6.0,0.2)^{T}$ and $\boldsymbol{c}^{(0)}=(6.0,1.0)^{T}$.

\subsubsection{Suboptimal adaptive controller}

The adaptive procedures considered in this section are based on algorithms originally introduced in [17] and [67]. Contrary to the TRPOD algorithm where the trust-region radius is revalued at each iteration, these authors consider the radius constant throughout the process of optimization $\left(\Delta^{(k+1)}=\right.$ $\Delta^{(k)}=\Delta$ ) and suppose that the range of validity of the POD ROM is independent of the specific control law used to derive it i.e. $\Delta=\infty$. However, since the flow dynamics depends a priori strongly on the control, it is not clear that a POD ROM, derived at iteration $k$ of the adaptive procedure, is suitable for describe the dynamics altered by the optimal control $\boldsymbol{c}^{(k+1)}$, solution of the reduced optimization problem at the next iteration. Therefore, it was suggested in [19] to modify the original algorithm while adding, in every iteration of the iterative procedure, the snapshots computed with the last optimal control input to the snapshot set used to determine the POD basis for the next iteration. The disadvantage of this procedure is that the size of the input data grows continuously with the iteration number, increasing considerably the computational costs. Hinze and Volkwein [19] successfully applied this modified algorithm to compute suboptimal controls for the cylinder wake flow at a Reynolds number equal to 100. Nevertheless, in the present case, after a few satisfying iterations, the control parameters reached erroneous values due to the divergence in the time integration of the POD ROM (12). This behavior expressed that the model (12), derived however with the control function method and POD basis functions with non-equilibrium modes, is not sufficiently robust to represent controlled dynamics located far from the different design operating conditions. It is thus necessary to restrict the range of validity of the POD ROM in the control parameter space in a way similar to what is made for the TRPOD algorithm. Various numerical values were considered for the parameters characteristic of this trust-region, the most satisfactory [56] were $\Delta_{A}=0.5$ and $\Delta_{S t_{f}}=0.1$ for the forcing amplitude and Strouhal num- 
ber respectively. Figure 9 represents for the different initial control $\boldsymbol{c}^{(0)}$, the variations of the values of the forcing amplitude and Strouhal number with respect to the iteration number. For $\boldsymbol{c}^{(0)}=(1.0,0.2)^{T}$, the forcing amplitude and Strouhal number oscillate around the values $A=3.25$ and $S t_{f}=0.65$ respectively. The corresponding mean drag coefficient is $\left\langle C_{D}\right\rangle_{T}=1.009$. For all the other values of initial parameters of control, the forcing amplitude and Strouhal number oscillate around $A=4.25$ and $S t_{f}=0.74$ respectively. In this case the value of the mean drag coefficient is $\left\langle C_{D}\right\rangle_{T}=0.993$. For the three last initial conditions, it is remarkable that the control parameters obtained by the adaptive procedure tend towards those given by numerical experimentation (see section 2.3). However, as one can note it on figure 9, the iterative process does not converge. Indeed, in the majority of the cases, the forcing amplitude oscillates constantly between the lower and the upper limits of the trust-region defined by $\Delta_{A}=0.5$. Since the global minimum of the mean drag coefficient is located in a very smooth valley (see Fig. 2), it is delicate to build a model function able to predict accurately the variations of the objective function in this area. The size of the trust-region $\Delta$ is then possibly too large at some iterations. It is thus necessary to envisage a mechanism of reduction of $\Delta$ during the optimization process in order to improve the robustness of the model functions. That is precisely the interest of the trust-region methods presented in section 3 .

\subsubsection{Optimal adaptive controller: the TRPOD approach}

According to the TRPOD algorithm $\mathrm{A}$, the radius of the trust-region $\Delta$ can now be automatically either increased, or decreased during the resolution of the optimization process. Essentially, the size of the trust-region depends on the topology of the objective function. Figure 10 represents for the different initial control vector $\boldsymbol{c}^{(0)}$, the variations of the values of the forcing amplitude and Strouhal number with respect to the iteration number. When the numerical convergence of the iterative procedure is achieved, the optimal control parameters are $A=4.25$ and $S t_{f}=0.738$. These values of parameters, which entirely define the optimal control law $\gamma_{\text {opt }}(t)$, are obtained in less than ten resolutions of the Navier-Stokes equations, whatever the initial condition considered (a more significant number of iterations is however represented in Fig. 10 to highlight the convergence). This convergence can be analyzed in more details while referring to [56] where the evolutions until convergence of the main parameters of the TRPOD algorithm are given. As it was expected by the global convergence properties of the TRPOD algorithm $(\S 3)$, these optimal control parameters tend towards the values predicted by an openloop control approach $(\S 2.3)$, and this, whatever the initial values used for the control parameters (see Fig. 11 which represents the convergence in the control parameter space). This proves the performance and the robustness of the TRPOD algorithm. Figure 12 represents the time evolutions of the aero- 

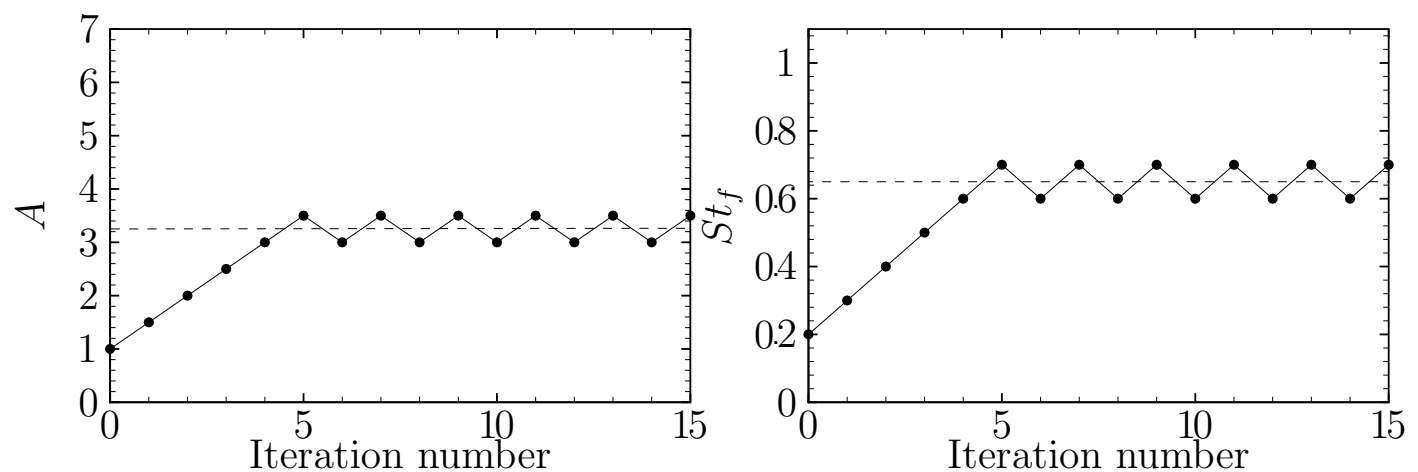

(a) $\boldsymbol{c}^{(0)}=(1.0,0.2)^{T}$.

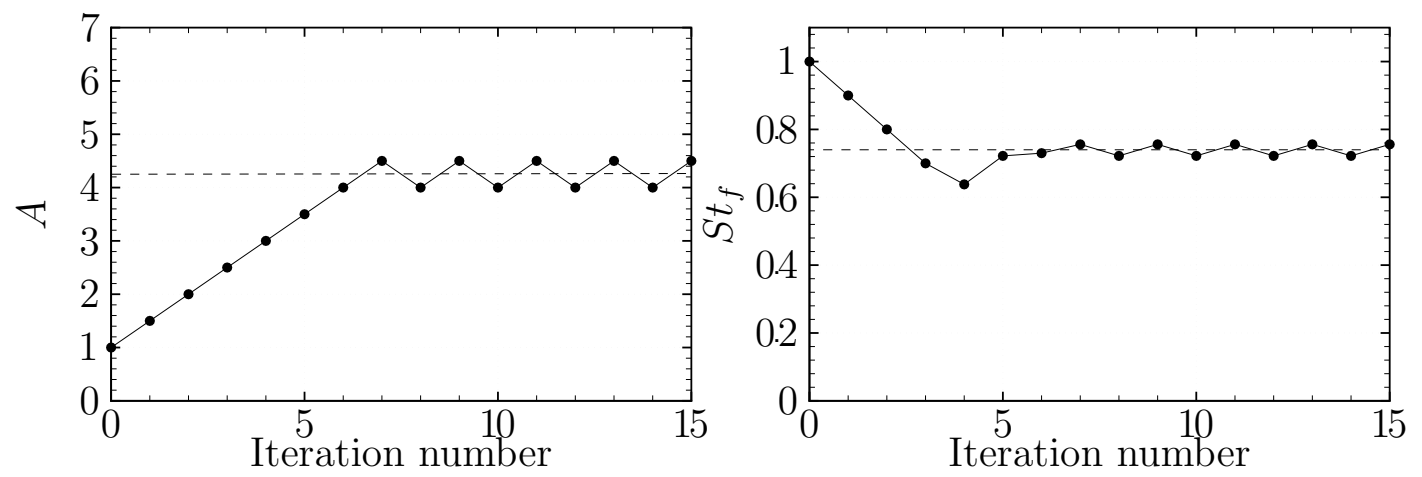

(b) $\boldsymbol{c}^{(0)}=(1.0,1.0)^{T}$.

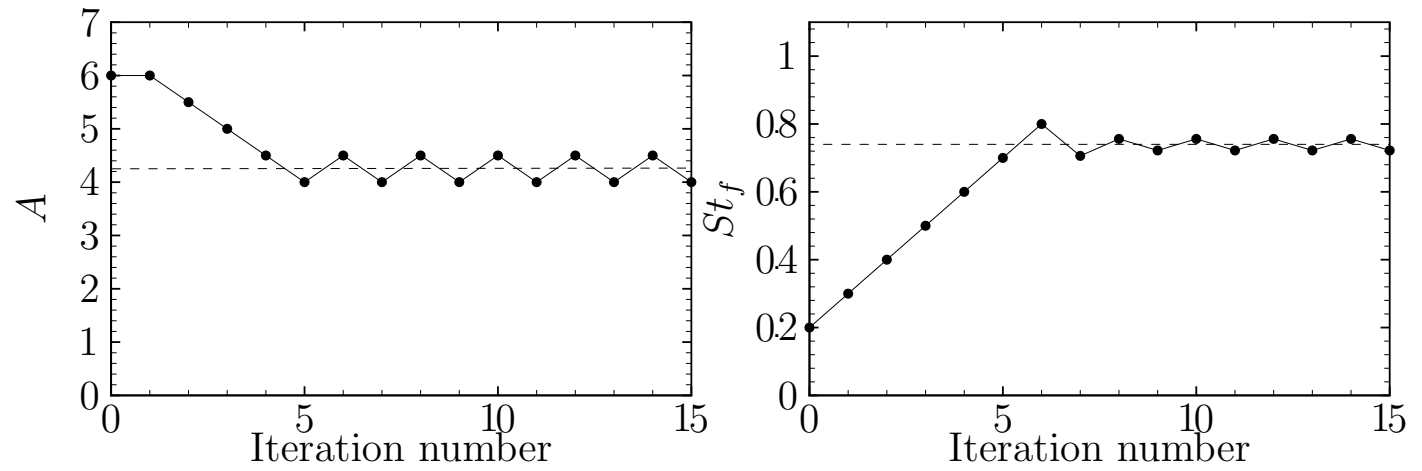

(c) $\boldsymbol{c}^{(0)}=(6.0,0.2)^{T}$.

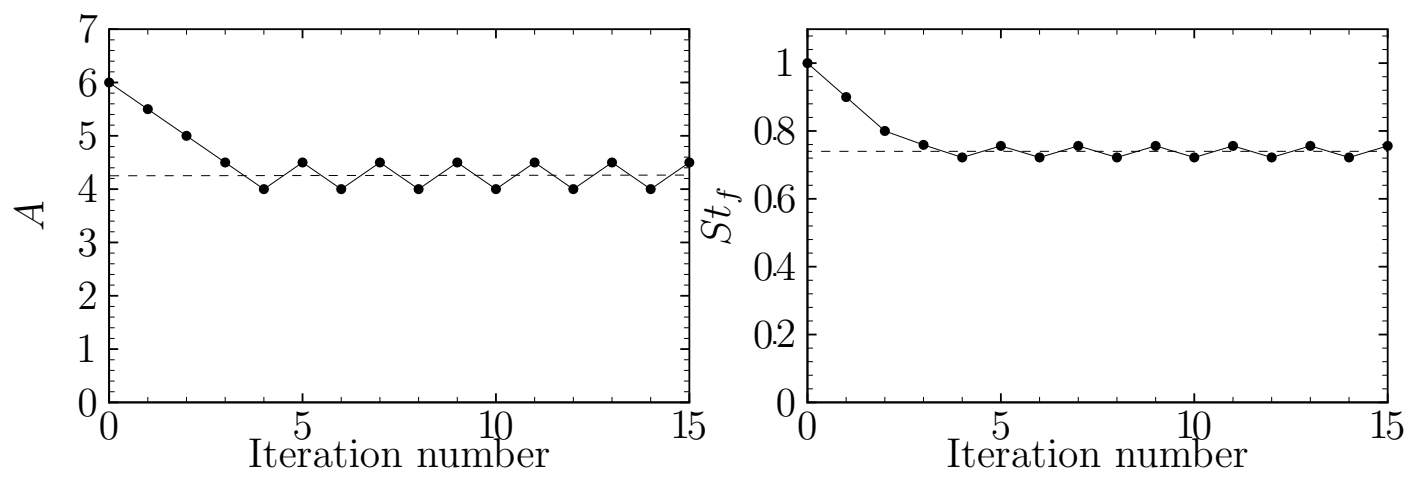

(d) $\boldsymbol{c}^{(0)}=(6.0,1.0)^{T}$.

Fig. 9. Variations of the forcing amplitude (left) and Strouhal number (right) with respect to the iteration number. Results obtained with the adaptive method for $\Delta_{A}=0.5$ and $\Delta_{S t_{f}}=0.1$. 

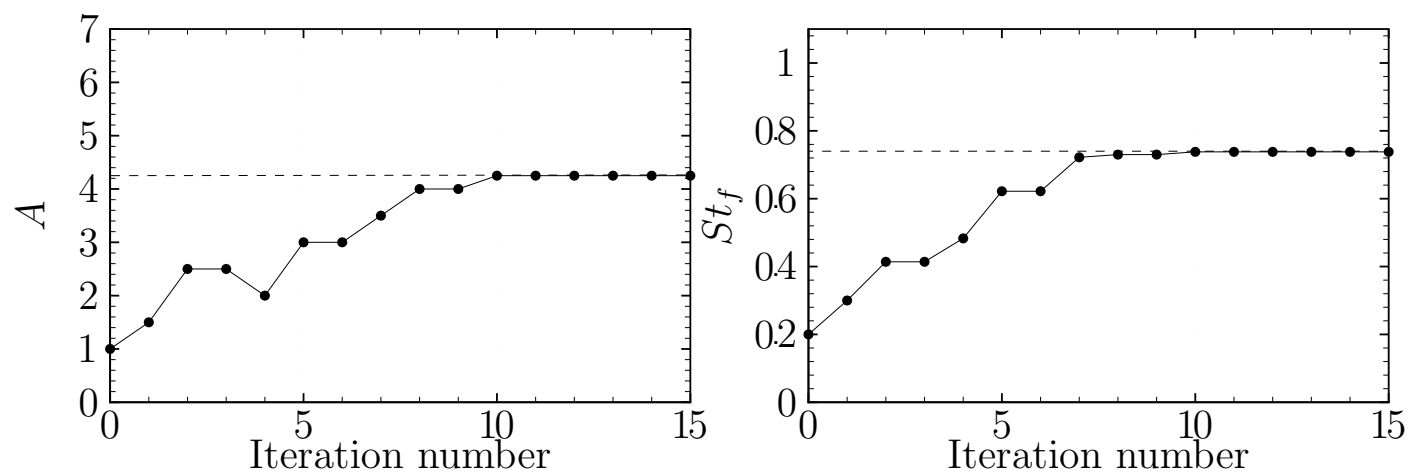

(a) $\boldsymbol{c}^{(0)}=(1.0,0.2)^{T}$.

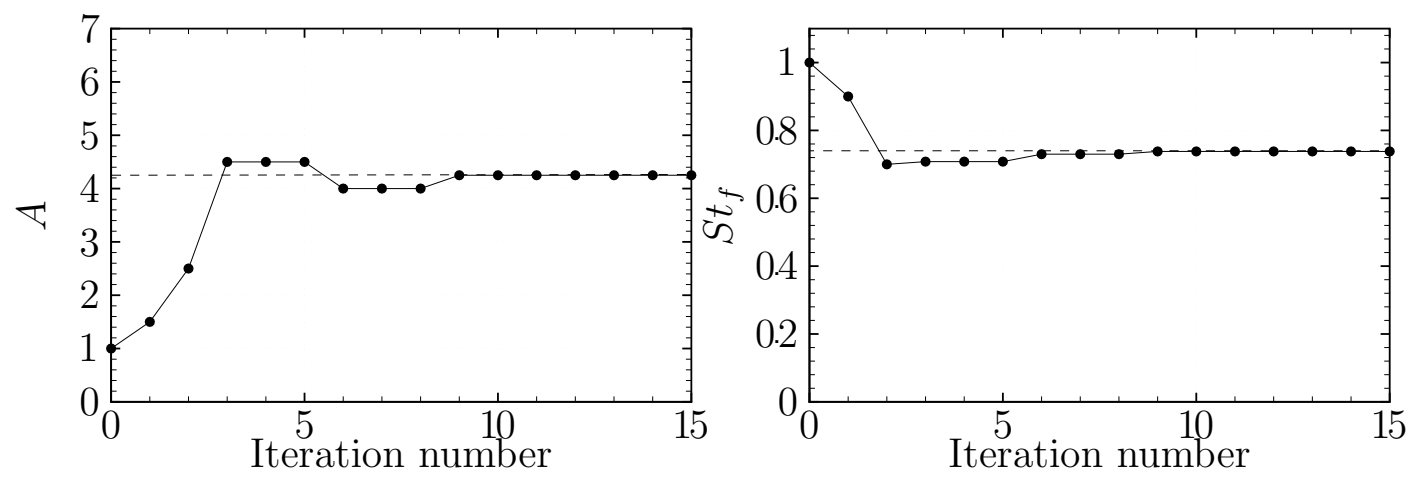

(b) $\boldsymbol{c}^{(0)}=(1.0,1.0)^{T}$.

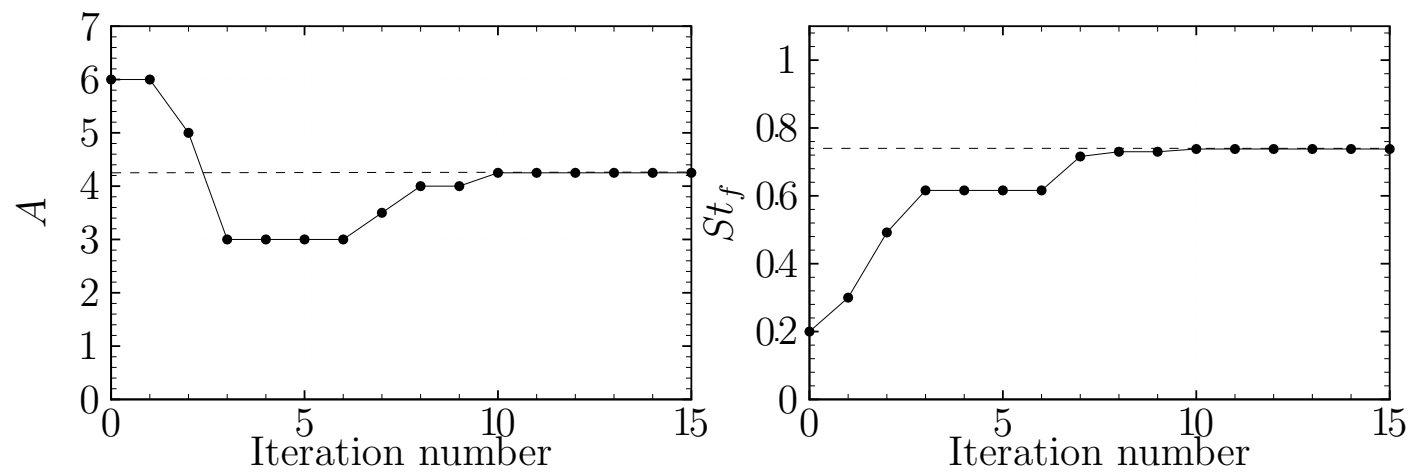

(c) $\boldsymbol{c}^{(0)}=(6.0,0.2)^{T}$.

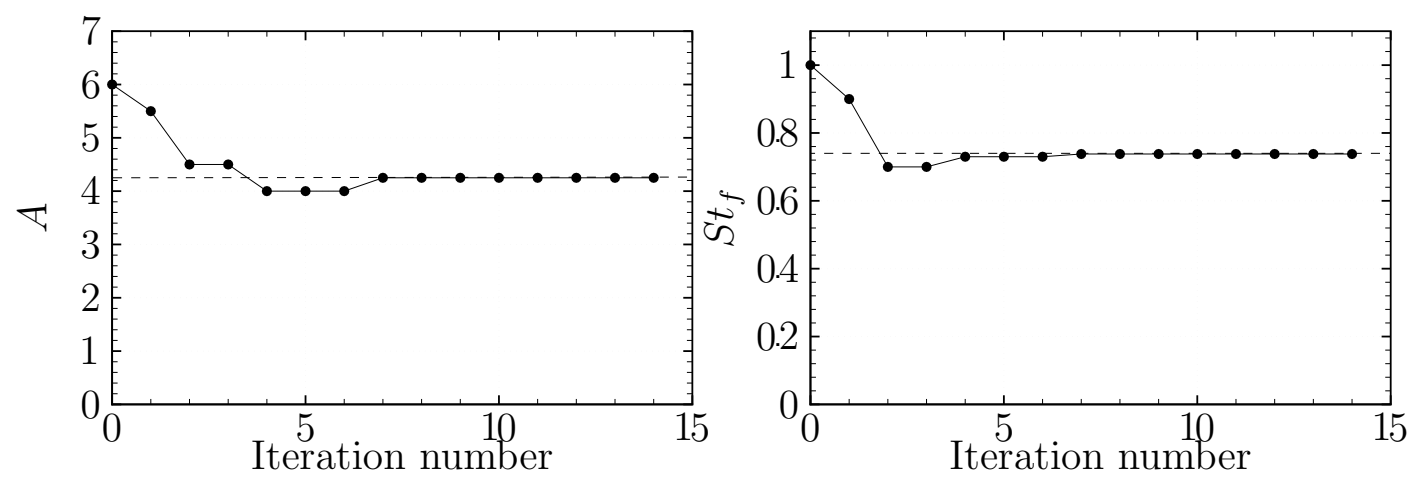

(d) $\boldsymbol{c}^{(0)}=(6.0,1.0)^{T}$.

Fig. 10. Variations of the forcing amplitude (left) and Strouhal number (right) with respect to the iteration number. Results obtained with the TRPOD method. 


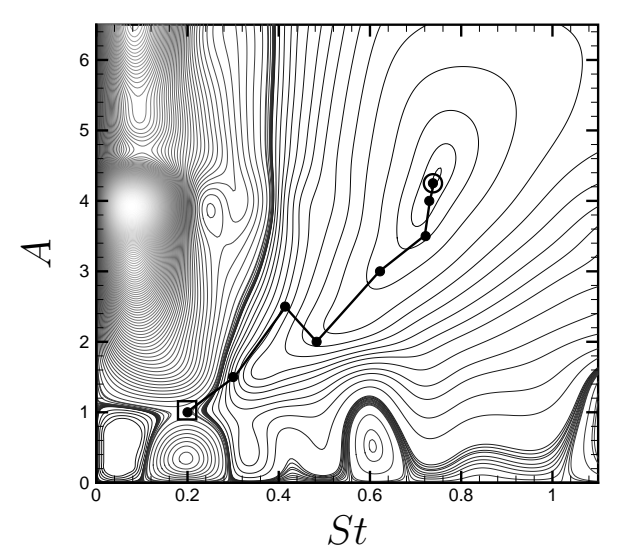

(a) $\boldsymbol{c}^{(0)}=(1.0,0.2)^{T}$

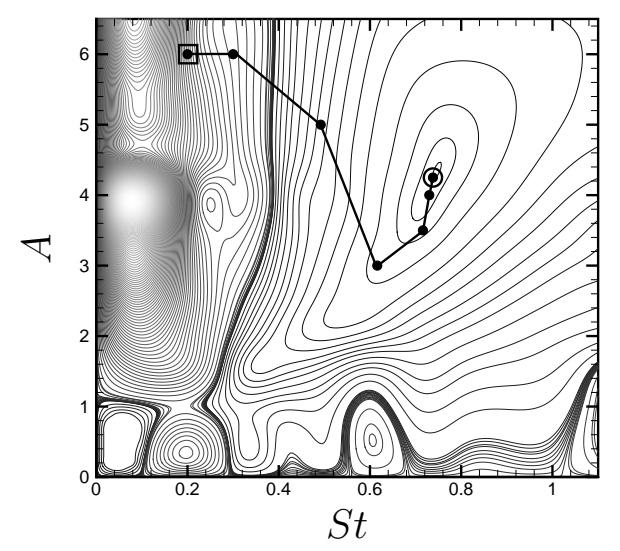

(c) $\boldsymbol{c}^{(0)}=(6.0,0.2)^{T}$

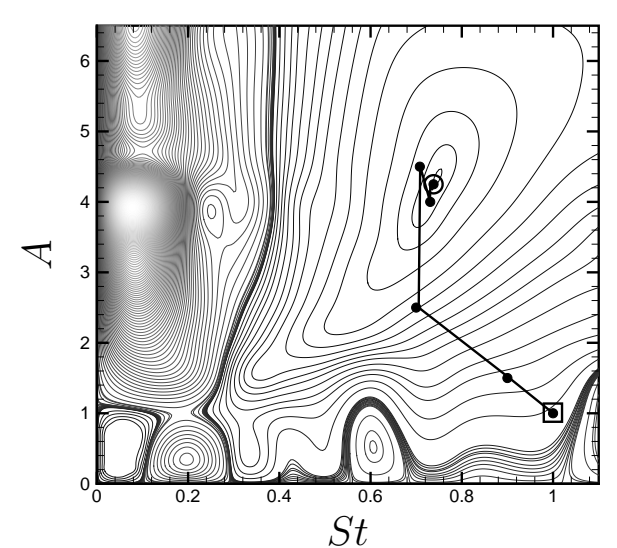

(b) $\boldsymbol{c}^{(0)}=(1.0,1.0)^{T}$

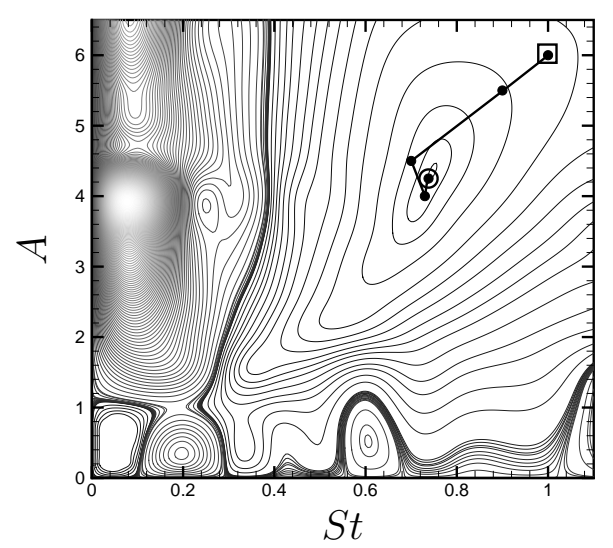

(d) $\boldsymbol{c}^{(0)}=(6.0,1.0)^{T}$

Fig. 11. Evolution of the control parameters during the optimization process for the different initial values $\boldsymbol{c}^{(0)}$.

dynamic coefficients, for an uncontrolled flow and for the flow forced by the optimal control law $\gamma_{\text {opt }}(t)$. These results are compared to those obtained for the unstable steady basic flow. It was argued in [30] that the basic flow generates a priori the lowest coefficient of drag for the configuration under study. The mean drag coefficient varies from a value equal to $\left\langle C_{D}{ }^{\text {unc }}\right\rangle_{T}=1.39$ in the uncontrolled case to a value equal to $\left\langle C_{D}{ }^{\text {opt }}\right\rangle_{T}=0.99$ when the optimal control parameters are applied. The corresponding relative mean drag reduction, defined as $\left(\left\langle C_{D}{ }^{\text {unc }}\right\rangle_{T}-\left\langle C_{D}{ }^{\text {opt }}\right\rangle_{T}\right) /\left\langle C_{D}{ }^{\text {unc }}\right\rangle_{T}$, is equal to more than $30 \%$. The value of the drag coefficient for the optimally controlled flow tends towards that obtained for the unstable steady basic flow $\left(C_{D}^{\text {basic }}=0.94\right)$, but with a value always slightly higher. In addition, similarly to the case of the uncontrolled flow [68], the drag coefficient oscillates at a frequency equal to twice that of the lift coefficient [56]. Furthermore, the controlled flow oscillates now at the frequency of the optimal control law $\left(S t_{f}=0.738\right)$, a phenomenon called as lock-on flow [56]. Finally, in Figs. 13(a)-13(c) we represent the vortic- 


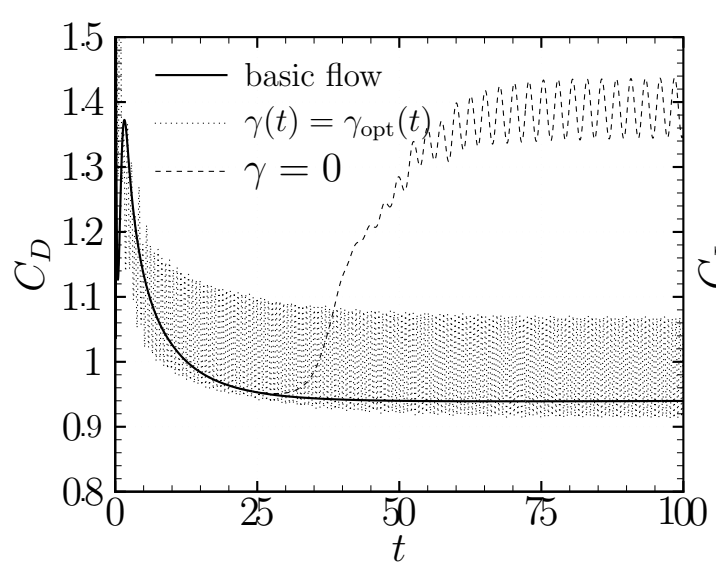

(a) Drag coefficient.

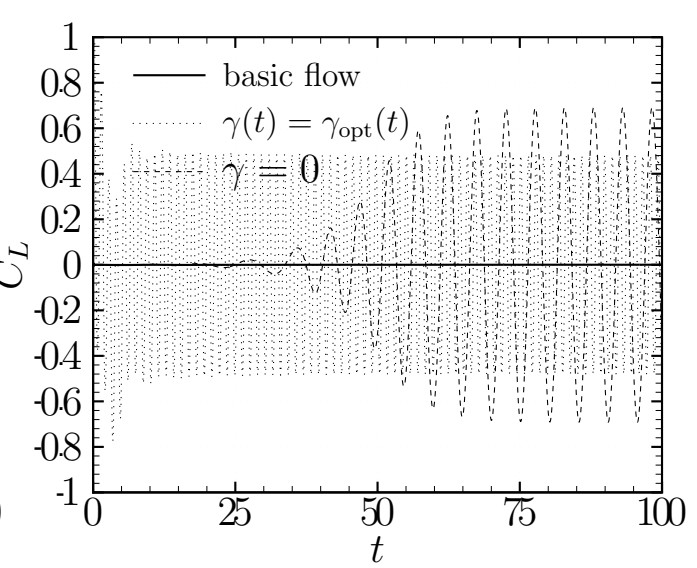

(b) Lift coefficient.

Fig. 12. Time evolutions of the aerodynamic coefficients for the basic flow (solid line), uncontrolled flow ( $\gamma=0$, dashed lines) and optimally controlled flow $\left(\gamma(t)=\gamma_{\text {opt }}(t)\right.$, dotted lines). Control was started at time $t=0$.

ity fields of the uncontrolled flow, the optimally controlled flow, and the basic flow, respectively. The significant vortex-shedding phenomenon observed in Fig. 13(a) has been substantially reduced when the control is applied and the flow has been quasisymmetrized. The resulting flow approaches the symmetric state characteristic of the corresponding basic flow as can be awaited from the results of [30] and the discussion in [15]. Our results are qualitatively similar to the effects observed in [21] and [38] and confirm the arguments of [40] that the mean drag reduction is associated with control driving the mean flow toward the unstable state.

\subsubsection{Discussion}

The numerical results obtained here with the TRPOD algorithm agree to a large extent to results obtained in other numerical approach, where the optimal control theory is applied for the same flow configuration directly to the Navier-Stokes equations (see table 1 for the characteristics of the different algorithms).

However, quantitative comparisons of the control algorithms presented in table 1 are difficult because for the comparisons to be fair, it would be necessary that the same actuation method and the same control objectives were retained in the various studies. But the studies used for comparison were performed with either a different actuation method or a different control objective. Therefore, only qualitative comparisons of the control methodologies are possible. The reader is referred to [15] for a detailed discussion.

Following [40], the energetic efficiency of the control can be characterized by 

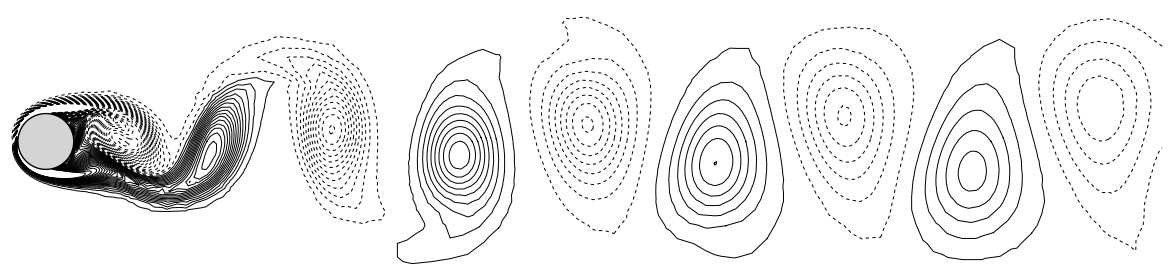

(a) Uncontrolled flow $(\gamma=0)$.

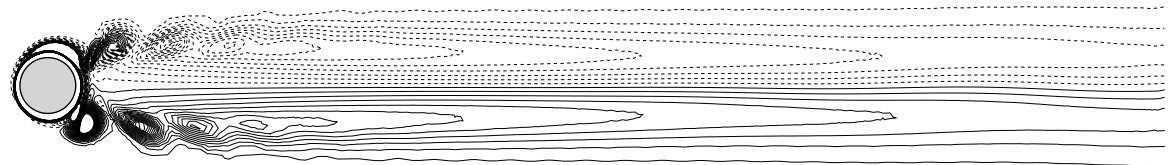

(b) Optimally controlled flow $(\gamma(t)=A \sin (2 \pi S t t)$ with $A=4.25$ and $S t=0.738)$.

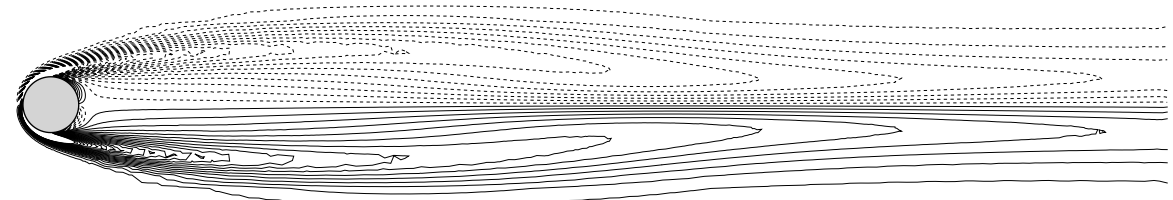

(c) Basic flow.

Fig. 13. Vorticity contour plot of the wake for the uncontrolled (a), optimally controlled (b) and basic flow (c). The dashed lines correspond to negative values.

the Power Saving Ratio (PSR) defined as:

$$
P S R=\frac{\left\langle P_{D}\right\rangle_{T}^{\text {uncontrolled }}-\left\langle P_{D}\right\rangle_{T}^{\text {controlled }}}{\left\langle P_{C}\right\rangle_{T}}
$$

where $\left\langle P_{D}\right\rangle_{T}$ and $\left\langle P_{C}\right\rangle_{T}$ represent respectively the mean of the instantaneous drag power $P_{D}$ and control power $P_{C}$ estimated over a finite horizon $T$. Except for the approach presented in [40] where the energetic efficiency is favored $(P S R \gg 1)$, the different numerical studies confirmed that an harmonic rotary control is energetically inefficient without a sufficient penalization of the control input. However, our main concern in this study is not to determine the control law with the maximum energetic efficiency. Rather, our objective is to demonstrate that with an appropriate adaptive strategy, the solution of the optimization problem based on POD reduced-order models of the flow corresponds to the solution of the optimization problem based on the Navier-Stokes 
Table 1

Characteristics of the different algorithms previously used in the literature to control the laminar wake flow with an optimal control approach. The present study is included for comparison. 'Unknown' means that the value was not found in the article, an estimate is then given when it is possible. In the column entitled "State equation", 'NS' means Navier-Stokes equations and 'POD ROM' means POD Reduced-Order Model. A similar table can be found in [15].

\begin{tabular}{|c|c|c|c|c|c|c|}
\hline Reference & Re & $\begin{array}{c}\text { Type of optimal } \\
\text { control law }\end{array}$ & State equation & Cost functional & $\begin{array}{l}\text { Relative mean } \\
\text { drag reduction }\end{array}$ & PSR \\
\hline$[38]$ & 200 & $\begin{array}{l}\text { Sinusoidal } \\
\qquad \begin{array}{c}A \\
S t_{f}\end{array}=0.75\end{array}$ & NS & Drag-related & $30 \%$ & $\begin{array}{c}\text { Unknown } \\
(\text { certainly }<1)\end{array}$ \\
\hline [39] & 100 & $\begin{array}{c}\text { Sinusoidal } \\
A=3.25 \\
S t_{f}=1.13\end{array}$ & NS & $\begin{array}{l}\text { Target flow } \\
\qquad(R e=2)\end{array}$ & Unknown & $\begin{array}{c}\text { Unknown } \\
(\text { certainly }<1)\end{array}$ \\
\hline [40] & 150 & Any & NS & $\begin{array}{c}\text { Power Drag } \\
+ \text { Power Control } \\
\end{array}$ & $15 \%$ & 51 \\
\hline$[15]$ & 200 & $\begin{array}{c}\text { Sinusoidal } \\
A=2.2 \\
S t_{f}=0.53\end{array}$ & POD ROM & Drag-related & $25 \%$ & 0.26 \\
\hline Present study & 200 & $\begin{array}{c}\text { Sinusoidal } \\
A=4.25 \\
S t=0.738\end{array}$ & POD ROM & Drag & $30 \%$ & 0.07 \\
\hline
\end{tabular}

equations. Therefore, the most outstanding result is that the optimal control parameters obtained by the TRPOD algorithm tend towards the parameters determined in section 2.3 by an open-loop control approach, thus confirming the results of global convergence of the TRPOD.

As it can be noticed in table 1, the relative drag reduction found with the Navier-Stokes equations as state equation is the same, sometimes slightly lower, than the one found with the TRPOD algorithm, but the numerical costs (CPU and memory) associated with their control are more important. Indeed, with a gradient-based algorithm of optimization, each iteration of the optimizer requires to determine a direction of descent (resolution of an optimality system formed by the state equations, the adjoint equations and the optimality conditions) then to carry out a line search which involves the resolution of several state equations (Navier-Stokes equations or POD ROM according to the adopted approach). Let us assume that in both cases the same numerical parameters are considered to solve the equations of the optimality system, that the convergence is obtained in the same number of iteration and, finally, that the CPU time necessary to solve the adjoint equations and the optimality conditions is the same that the one used for the state equations. In this study, the CPU time necessary to obtain, with the POD ROM, the flow dynamics over a given time horizon represents $1 \%$ of the time corresponding to the resolution of the Navier-Stokes equations with the finite-element approach. 
Consequently, the computational time necessary to solve the optimality system based on the POD ROM can be neglected at first approximation in front of the CPU time necessary to generate the POD ROM. The computational costs related to the resolution of the optimality system based on the Navier-Stokes equations are at least approximately equal to four times that required to solve the optimization problem based on the POD ROM [52]. This cost corresponds to the case where only one optimality condition and one line search step are considered. Consequently, in practice, the reduction factor can be largely more important and that, even if additional resolutions of the Navier-Stokes equations are necessary, to evaluate the non-equilibrium modes used to build the POD basis.

With regard to memory cost, note that we need to store the state variables for all space time to solve the adjoint equations and all the adjoint variables to estimate the optimality conditions. When the finite-element simulation is used to solve the optimal control problem over a time horizon $T_{o}$, we need to store the state and adjoint variables (two velocity components and the pressure) at every time step and for each vertex of the mesh. When the POD ROM is used, we only need to store the time evolution of a and of the adjoint variables $\boldsymbol{\xi}$ for $N_{\text {gal }}^{r}+N_{\text {neq }}+1$ POD modes plus the coefficients appearing in the state equation $(12)$ i.e. four linear coefficients $(\mathcal{A}, \mathcal{D}, \mathcal{E}$ and $\mathcal{G})$, two quadratic coefficients $(\mathcal{B}$ and $\mathcal{F})$ and one cubic coefficient $(\mathcal{C})$. As illustration, we consider that the parameters used to solve the optimization problem are $T_{o}=20$ for the time horizon, $\Delta t=0.01$ for the optimization time-step, $N_{v}=12,000$ for the number of vertices and $N_{\text {gal }}^{r}+N_{\text {neq }}+1=20$ for the number of POD modes kept in the ROM where $N_{\text {gal }}^{r}=14$ corresponds in this case to $99.9 \%$ of the Relative Information Content. We found after estimate [52] that the memory cost of the POD ROM approach is approximately 1600 times lesser than for the Navier-Stokes model (approximately 180 if we decide to store the POD eigenfunctions to reconstruct later the velocity and pressure fields). The reduction of the numerical costs offered by our approach is so important that the study of three-dimensional unsteady complex flows by the optimal control theory becomes possible. The cost-reduction factors are comparable to the results previously found in [15] but, in this case, the global convergence of the TRPOD algorithm can be used to prove mathematically that the iterations produced by the otptimization algorithm will converge to a local optimizer for the high-fidelity model.

\section{Conclusions}

The objective of this paper was to demonstrate the applicability and computational savings which can be offered by combining trust-region methods and POD Reduced-Order Models to solve an optimal control problem for 
fluid flows. The Trust-Region POD algorithm originally introduced in [50] was used to minimize the total mean drag coefficient of a circular cylinder wake flow in the laminar regime $(R e=200)$. Since the cost functional is the mean drag, the POD basis functions were extended to the pressure data. A particular care was taken to derive a POD ROM for the pressure and velocity fields with an appropriate balance between model accuracy and robustness. The key enablers are the calibration of the POD ROM by the introduction of an optimal eddy-viscosity for each POD mode and the addition in the POD expansion of several non-equilibrium modes to describe various operating conditions. Finally, the optimal control parameters obtained with the TRPOD algorithm are $A=4.25$ and $S t_{f}=0.738$. The relative mean drag reduction is equal to $30 \%$ : the mean drag coefficient varies from a value equal to 1.39 in the uncontrolled case to a value equal to 0.992 when the optimal control parameters are applied. However, we demonstrated that this control law is energetically inefficient. These numerical results agree to a large extent to those obtained previously by other researchers $[38,40,69]$ using the twodimensional Navier-Stokes equations to solve the optimal control problem. Compared with those studies, the main advantage of our approach is that it leads to a significant reduction of the numerical costs because the optimization process itself is completely based on reduced-order models only. Indeed, when the state equations of the optimality system are POD ROMs instead of the Navier-Stokes equations, a cost reduction factor of 1600 is obtained for the memory and the optimization problem is solved approximately 4 times more quickly. Now, if we compare to our preceding study [15], where a POD ROM was coupled to an optimal control approach without any strategy for updating the reduced-order model during the optimization process, the cost reduction factors, found here, are lower. However, in this study, the use of the TRPOD algorithm mathematically proves that the solutions converge at least to a local optimum for the original high-fidelity problem, and less than ten resolutions of the Navier-Stokes equations are necessary. Due to the low computational costs involved in the optimization process and the mathematical proofs of global convergence, the TRPOD algorithm is a promising method of optimization in flow control. This approach that can easily be adapted to other configurations, should finally lead to the current resolution of unsteady, three-dimensional optimization problems for turbulent flows around complex geometries.

\section{Acknowledgements}

The authors acknowledge M. Braza (Institut de Mécanique des Fluides de Toulouse) and D. Ruiz (ENSEEIHT), who kindly provided an original version of their Matlab Navier-Stokes solver. Stimulating and fruitful discussions with 
Jean-Pierre Brancher and the low-dimensional modelling and control team at the Technische Universität Berlin, in particular Bernd R. Noack, are acknowledged.

\section{A The Trust-Region Proper Orthogonal Decomposition algorithm}

This appendix describes the TRPOD algorithm used in section 5.2.2.

TRPOD ALGORITHM. Initialization: Let $\eta_{1}, \eta_{2}, \gamma_{1}, \gamma_{2}$ and $\gamma_{3}$ be five positive constants such as $0<\eta_{1}<\eta_{2}<1$ and $0<\gamma_{1} \leq \gamma_{2}<1 \leq \gamma_{3}$. Let $\Delta^{(0)}>0$ be an initial trust-region radius and $\boldsymbol{c}^{(0)}$ the initial control vector. Compute a set of snapshots $\mathcal{U}^{(0)}$ corresponding to the control $\boldsymbol{c}^{(0)}$ and estimate the value of the objective function $f\left(\boldsymbol{c}^{(0)}\right)$. Set $k=0$.

(1) Compute a POD basis $\left\{\boldsymbol{\phi}_{i}^{(k)}\right\}_{i=1, \ldots, N_{\mathrm{POD}}}$ using the snapshots $\mathcal{U}^{(k)}$ and derive a POD ROM of the controlled flow.

(2) Compute the model function $m^{(k)}$ and solve the sub-problem

$$
\boldsymbol{s}^{(k)}=\arg \min _{\boldsymbol{s} \in \mathbb{R}^{n}} m^{(k)}\left(\boldsymbol{c}^{(k)}+\boldsymbol{s}\right) \quad \text { subject to }\|\boldsymbol{s}\| \leq \Delta^{(k)} .
$$

(3) Compute a snapshot set $\mathcal{U}^{(k+)}$ corresponding to the control $\boldsymbol{c}^{(k)}+\boldsymbol{s}^{(k)}$ and estimate the value of the objective function $f\left(\boldsymbol{c}^{(k)}+\boldsymbol{s}^{(k)}\right)$. Determine $\rho^{(k)}$ :

$$
\rho^{(k)}=\frac{f\left(\boldsymbol{c}^{(k)}+\boldsymbol{s}^{(k)}\right)-f\left(\boldsymbol{c}^{(k)}\right)}{m\left(\boldsymbol{c}^{(k)}+\boldsymbol{s}^{(k)}\right)-m\left(\boldsymbol{c}^{(k)}\right)} .
$$

(4) Update the trust-region radius:

- If $\rho^{(k)} \geq \eta_{2}$, the step is accepted: the iteration is successful. Set $\boldsymbol{c}^{(k+1)}=$ $\boldsymbol{c}^{(k)}+\boldsymbol{s}^{(k)}, \mathcal{U}^{(k+1)}=\mathcal{U}^{(k+)}$ and choose $\Delta^{(k+1)} \in\left[\Delta^{(k)}, \gamma_{3} \Delta^{(k)}\right]$. If a given criterion of convergence is verified, the algorithm is stopped, else, set $k=k+1$ and return at the stage (1).

- If $\eta_{1} \leq \rho^{(k)}<\eta_{2}$, the step is accepted: the iteration is successful. Set $\boldsymbol{c}^{(k+1)}=\boldsymbol{c}^{(k)}+\boldsymbol{s}^{(k)}, \mathcal{U}^{(k+1)}=\mathcal{U}^{(k+)}$ and choose $\Delta^{(k+1)} \in\left[\gamma_{2} \Delta^{(k)}, \Delta^{(k)}\right]$. If a given criterion of convergence is verified, the algorithm is stopped, else, set $k=k+1$ and return at the stage (1).

- If $\rho^{(k)}<\eta_{1}$, the step is refused: the iteration is unsuccessful. Set $\boldsymbol{c}^{(k+1)}=$ $\boldsymbol{c}^{(k)}, \mathcal{U}^{(k+1)}=\mathcal{U}^{(k)}$ and choose $\Delta^{(k)} \in\left[\gamma_{1} \Delta^{(k)}, \gamma_{2} \Delta^{(k)}\right]$. Set $k=k+1$ and return at the stage (2).

The usual parameters for the TRPOD algorithm are $\eta_{1}=0.25$ and $\eta_{2}=0.75$ for the criteria of performance, and $\gamma_{1}=0.25, \gamma_{2}=0.75$ and $\gamma_{3}=2$ for the criteria of actualization [50]. 


\section{References}

[1] M. D. Gunzburger, Flow control, Springer, New York, 1995.

[2] M. D. Gunzburger, Introduction into mathematical aspects of flow control and optimization, in: Lecture series 1997-05 on inverse design and optimization methods, Von Kármán Institute for Fluid Dynamics, 1997.

[3] M. D. Gunzburger, Adjoint Equation-Based Methods for Control Problems in Incompressible, Viscous Flows, Flow, Turbulence and Combustion 65 (2000) 249-272.

[4] A. J. Booker, J. E. Dennis Jr., P. D. Frank, D. B. Serafini, V. Torczon, M. W. Trosset, A rigorous framework for optimization of expensive functions by surrogates, Structural Optimization 17 (1) (1999) 1-13.

[5] A. C. Antoulas, Approximation of Large-Scale Dynamical Systems, SIAM, 2005.

[6] K. Ito, S. S. Ravindran, A reduced-order method for simulation and control of fluid flows, J. Comp. Phys. 143 (1998) 403-425.

[7] J. L. Lumley, Atmospheric Turbulence and Wave Propagation. The structure of inhomogeneous turbulence, A.M. Yaglom \& V.I. Tatarski, 1967, pp. 166-178.

[8] L. Sirovich, Turbulence and the dynamics of coherent structures, Quarterly of Applied Mathematics XLV (3) (1987) 561-590.

[9] K. E. Willcox, Reduced-order aerodynamic models for aeroelastic control of turbomachines, Ph.D. thesis, Massachusetts Institute of Technology (2000).

[10] J. Burkardt, M. D. Gunzburger, H.-C. Lee, Centroidal Voronoi TessellationBased Reduced-Order Modeling of Complex Systems, Tech. rep., Florida State University (2004).

[11] C. W. Rowley, Model reduction for fluids, using balanced proper orthogonal decomposition, Int. J. on Bifurcation and Chaos 15 (3) (2005) 997-1013.

[12] X. Ma, G. E. Karniadakis, A low-dimensional model for simulating threedimensional cylinder flow, J. Fluid Mech. 458 (2002) 181-190.

[13] B. R. Noack, K. Afanasiev, M. Morzyński, G. Tadmor, F. Thiele, A hierarchy of low-dimensional models for the transient and post-transient cylinder wake, J. Fluid Mech. 497 (2003) 335-363.

[14] B. R. Noack, G. Tadmor, M. Morzyński, Low-dimensional models for feedback flow control. Part I: Empirical Galerkin models, in: 2nd AIAA Flow Control Conference, Portland, Oregon, U.S.A., June 28 - July 1, 2004, AIAA-Paper 2004-2408 (invited contribution).

[15] M. Bergmann, L. Cordier, J.-P. Brancher, Optimal rotary control of the cylinder wake using POD Reduced Order Model, Phys. Fluids 17 (9) (2005) 097101:1-21. 
[16] W. R. Graham, J. Peraire, K. T. Tang, Optimal Control of Vortex Shedding Using Low Order Models. Part 1. Open-Loop Model Development, Int. J. for Numer. Meth. in Engrg. 44 (7) (1999a) 945-972.

[17] S. S. Ravindran, Reduced-order adaptive controllers for fluid flows using POD, J. of Scientific Computing 15 (4) (2000) 457-478.

[18] S. S. Ravindran, Adaptive Reduced-Order Controllers for a Thermal Flow System, SIAM Journal on Scientific Computing 23 (6) (2002) 1925-1943.

[19] M. Hinze, S. Volkwein, Proper Orthogonal Decomposition Surrogate Models for Nonlinear Dynamical Systems: Error Estimates and Suboptimal Control, Tech. rep., Preprint SFB609, Technische Universität Dresden (2004).

[20] C. H. K. Williamson, Vortex dynamics in the cylinder wake, Ann. Rev. Fluid. Mech. 28 (1996) 477-539.

[21] P. T. Tokumaru, P. E. Dimotakis, Rotary oscillatory control of a cylinder wake, J. Fluid Mech. 224 (1991) 77-90.

[22] X.-Y. Lu, J. Sato, A numerical study of flow past a rotationally oscillating circular cylinder, J. Fluids Struct. 10 (1996) 829-849.

[23] M. H. Chou, Synchronization of vortex shedding from a cylinder under rotary oscillation, Computers \& Fluids 26 (1997) 755-774.

[24] S. J. Baek, H. J. Sung, Numerical simulation of the flow behind a rotary oscillating circular cylinder, Phys. Fluids 10 (4) (1998) 869-876.

[25] F. M. Mahfouz, H. M. Badr, Flow Structure in the Wake of a Rotationally Oscillating Cylinder, Journal of Fluids Engineering 122 (2) (2000) 290-301.

[26] S.-J. Baek, H. J. Sung, Quasi-periodicity in the wake of a rotationally oscillating cylinder, J. Fluid Mech. 408 (2000) 275-300.

[27] M. Cheng, Y. T. Chew, S. C. Luo, Numerical investigation of a rotationally oscillating cylinder in mean flow, J. Fluids Struct. 15 (2001) 981-1007.

[28] M. Cheng, G. R. Liu, K. Y. Lam, Numerical simulation of flow past a rotationally oscillating cylinder, Computers \& Fluids 30 (2001) 365-392.

[29] S. Choi, H. Choi, S. Kang, Characteristics of flow over a rotationally oscillating cylinder at low Reynolds number, Phys. Fluids 14 (8) (2002) 2767-2777.

[30] B. Protas, J.-E. Wesfreid, Drag force in the open-loop control of the cylinder wake in the laminar regime, Phys. Fluids 14 (2) (2002) 810-826.

[31] N. Fujisawa, K. Ikemoto, K. Nagaja, Vortex shedding resonnance from a rotationally oscillating cylinder, J. Fluids Struct. 12 (1998) 1041-1053.

[32] N. Fujisawa, Y. Kawaji, K. Ikemoto, Feedback control of vortex shedding from a circular cylinder by rotational oscillations, J. Fluids Struct. 15 (2001) 23-37. 
[33] S. Goujon-Durand, J.-E. Wesfreid, P. Jenffer, Contrôle actif du sillage autour d'un cylindre oscillant, in: 15th French Congress of Mechanics, Nancy, Sept. 3 $-7,2001$.

[34] B. Thiria, S. Goujon-Durand, J.-E. Wesfreid, Wake of a cylinder performing rotary oscillations, Submitted to J. Fluid Mech.

[35] F. Abergel, R. Temam, On some control problems in fluid mechanics, Theoret. Comput. Fluid Dynamics 1 (1990) 303.

[36] T. R. Bewley, Flow control: new challenges for a new Renaissance, Progress in Aerospace Sciences 37 (2001) 21-58.

[37] T. R. Bewley, The emerging roles of model-based control theory in fluid mechanics, in: Advances in Turbulence IX, 2002, ninth European Turbulence Conference.

[38] J.-W. He, R. Glowinski, R. Metcalfe, A. Nordlander, J. Périaux, Active control and drag optimization for flow past a circular cylinder. Part 1. Oscillatory cylinder rotation, J. Comp. Phys. 163 (2000) 83-117.

[39] C. Homescu, I. M. Navon, Z. Li, Suppression of vortex shedding for flow around a circular cylinder using optimal control, Int. J. Numer. Meth. Fluids 38 (2002) $43-69$.

[40] B. Protas, A. Styczek, Optimal rotary control of the cylinder wake in the laminar regime, Phys. Fluids 14 (7) (2002) 2073-2087.

[41] T. R. Bewley, P. Moin, R. Temam, DNS-based predictive control of turbulence: an optimal benchmark for feedback algorithms, J. Fluid Mech. 447 (2001) 179225 .

[42] Y. Chang, Approximate models for optimal control of turbulent channel flow, Ph.D. thesis, Rice university (2000).

[43] M. Wei, J. B. Freund, A noise-controlled free shear flow, J. Fluid Mech. 546 (2006) $123-152$.

[44] N. Alexandrov, J. E. Dennis Jr, R. M. Lewis, V. Torczon, A Trust Region framework for managing the use of approximation models in optimization, Icase report 97-50.

[45] M. Bergmann, L. Cordier, J.-P. Brancher, On the power used to control the circular cylinder drag by rotary oscillations, Phys. Fluids 18 (8) (2006) 088103:1-4.

[46] B. R. Noack, H. Eckelmann, A global stability analysis of the steady and periodic cylinder wake, J. Fluid Mech. 270 (1994) 297-330.

[47] D. Barkley, R. D. Henderson, Three-dimensional Floquet stability analysis of the wake of a circular cylinder, J. Fluid Mech. 322 (1996) 215-241. 
[48] L. Cordier, M. Bergmann, Proper Orthogonal Decomposition: an overview, in: Lecture series 2002-04 on post-processing of experimental and numerical data, Von Kármán Institute for Fluid Dynamics, 2002.

[49] W. R. Graham, J. Peraire, K. T. Tang, Optimal Control of Vortex Shedding Using Low Order Models. Part 2: Model-based control, Int. J. for Numer. Meth. in Engrg. 44 (7) (1999b) 973-990.

[50] M. Fahl, Trust-region methods for flow control based on Reduced Order Modeling, Ph.D. thesis, Trier university (2000).

[51] J. Nocedal, S. J. Wright, Numerical Optimization, Springer series in operations research, 1999.

[52] M. Bergmann, L. Cordier, Optimal flow control of the cylinder wake by POD Reduced Order Models and Trust-Region methods, Tech. rep., INRIA (to appear in 2008).

[53] E. Arian, M. Fahl, E. W. Sachs, Trust-Region Proper Orthogonal Decomposition for Flow Control, Icase report 2000-25.

[54] A. R. Conn, N. I. M. Gould, P. L. Toint, Trust-region methods, SIAM, Philadelphia, 2000.

[55] P. L. Toint, Global convergence of a class of trust-region methods for nonconvex minimization in Hilbert space, IMA J. Numer. Anal. 8 (2) (1988) 231-252.

[56] M. Bergmann, Optimisation aérodynamique par réduction de modèle POD et contrôle optimal. Application au sillage laminaire d'un cylindre circulaire., Ph.D. thesis, Institut National Polytechnique de Lorraine, Nancy, France (2004).

[57] B. R. Noack, P. Papas, P. A. Monkewitz, The need for a pressure-term representation in empirical galerkin models of incompressible shear-flows, J. Fluid Mech. 523 (2005) 339-365.

[58] B. Galletti, C.-H. Bruneau, L. Zannetti, A. Iollo, Low-order modelling of laminar flow regimes past a confined square cylinder, J. Fluid Mech. 503 (2004) $161-170$.

[59] C. Kasnakoglu, Reduced order modeling, nonlinear analysis and control methods for flow control problems, Ph.D. thesis, Ohio State University (2007).

[60] H. Gunes, S. Sirisup, G. E. Karniadakis, Gappy data: To Krig or not to Krig?, J. Comp. Phys. 212 (2006) 358-382.

[61] K. Willcox, Unsteady Flow Sensing and Estimation via the Gappy Proper Orthogonal Decomposition, Computers and Fluids 35 (2) (2006) 208-226.

[62] D. Rempfer, H. F. Fasel, Evolution of three-dimensional coherent structures in a flat-plate boundary layer, J. Fluid Mech. 260 (1994) 351-375.

[63] M. Couplet, C. Basdevant, P. Sagaut, Calibrated reduced-order POD-Galerkin system for fluid flow modelling, J. Comp. Phys. 207 (2005) 192-220. 
[64] B. Galletti, A. Bottaro, C.-H. Bruneau, A. Iollo, Accurate model reduction of transient and forced wakes, Eur. J. Mech. B/Fluids 26 (3) (2007) 354-366.

[65] J. Favier, L. Cordier, A. Kourta, Accurate POD Reduced-Order Models of separated flows, Phys. Fluids (Submitted for publication).

[66] A. E. Deane, I. G. Kevrekidis, G. E. Karniadakis, S. A. Orszag, Low-dimensional models for complex geometry flows: Application to grooved channels and circular cylinders, Phys. Fluids 3 (10) (1991) 2337-2354.

[67] K. Afanasiev, M. Hinze, Adaptive control of a wake flow using Proper Orthogonal Decomposition, in: Shape Optimization and Optimal Design, Lecture Notes in Pure and Applied Mathematics, Vol. 216, Marcel Dekker, 2001.

[68] B. Protas, J.-E. Wesfreid, On the relation between the global modes and the spectra of drag and lift in periodic wake flows, C.R. Mécanique 331 (2003) $49-54$.

[69] C. Homescu, L. R. Petzold, R. Serban, Error Estimation for Reduced Order Models of Dynamical Systems, Tech. rep., Lawrence Livermore National Laboratory (2003). 